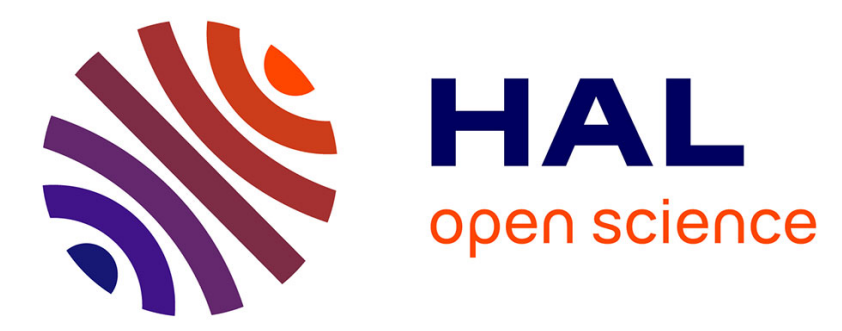

\title{
Wavelet-Based Separation Methods Assessment on the Near Pressure Field of a Landing Gear Subcomponent
} Antoine Hajczak, Laurent Sanders, Vuillot François, Philippe Druault

\section{To cite this version:}

Antoine Hajczak, Laurent Sanders, Vuillot François, Philippe Druault. Wavelet-Based Separation Methods Assessment on the Near Pressure Field of a Landing Gear Subcomponent. 25th AIAA/CEAS Aeroacoustic Conference, May 2019, DELFT, Netherlands. 10.2514/6.2019-2482 . hal-02140504

\section{HAL Id: hal-02140504 https://hal.sorbonne-universite.fr/hal-02140504}

Submitted on 27 May 2019

HAL is a multi-disciplinary open access archive for the deposit and dissemination of scientific research documents, whether they are published or not. The documents may come from teaching and research institutions in France or abroad, or from public or private research centers.
L'archive ouverte pluridisciplinaire HAL, est destinée au dépôt et à la diffusion de documents scientifiques de niveau recherche, publiés ou non, émanant des établissements d'enseignement et de recherche français ou étrangers, des laboratoires publics ou privés. 


\title{
Wavelet-Based Separation Methods Assessment on the Near Pressure Field of a Landing Gear Subcomponent
}

\author{
Antoine Hajczak*, Laurent Sanders ${ }^{\dagger}$, François Vuillot ${ }^{\star}$ \\ ONERA - The French Aerospace Lab, F-92322 Châtillon, France \\ Philippe Druault ${ }^{\S}$ \\ Sorbonne Université, CNRS UMR 7190, \\ Institut Jean le Rond d'Alembert, F-75005 Paris, France
}

\begin{abstract}
The pressure field in the vicinity of a shear layer developing over the circular cavity installed in a landing gear wheel is investigated numerically by means of wavelet-based filtering algorithms originally developed for the analysis of turbulent jet noise by Mancinelli et al. $(J$. Fluid Mech. (2017), vol. 813, pp. 716-749). First, the well-known wavenumber-frequency filtering in the Fourier space is performed on a linear array of microphones over the shear layer. Then, four separation algorithms are successively tested at a given observer location on this array. We show that in the specific case of CFD signals, when the mesh cut-off frequency is lower than the Nyquist frequency, the recursive filtering method only extracts the background numerical noise of the simulation due to the band-pass filter behavior of the wavelet daughters at the smallest scales. Resampling the data yields more physical results by suppressing the Gaussian but nonphysical noise of the signal. Overall, important scatter is found between the methods in the low frequency range. An analysis of the filtered pressure decay with distance is finally carried out on the basis of expected near and far-field behavior of the acoustic field.
\end{abstract}

\section{Nomenclature}

\begin{tabular}{|c|c|c|c|c|c|}
\hline$\left(\vec{e}_{x}, \vec{e}_{y}, \vec{e}_{z}\right)$ & $=$ & Cartesian reference frame & $(x, y, z)$ & $=$ & Spatial coordinates \\
\hline$L$ & $=$ & Pressure field reference length & $D_{w}$ & $=$ & Wheel diameter \\
\hline$H_{w}$ & $=$ & Wheel thickness & $R=0.27 D_{w}$ & $=$ & Cavity radius \\
\hline$\kappa=\frac{H}{2 R}$ & $=$ & Cavity depth-to-diameter ratio & $U_{\infty}$ & $=$ & Free-stream velocity \\
\hline$c_{0}$ & $=$ & Speed of sound & $M=\frac{U_{\infty}}{c_{0}}$ & $=$ & Free-stream Mach number \\
\hline$y^{+}$ & $=$ & Dimensionless wall distance & $f \quad c^{0}$ & $=$ & Frequency \\
\hline$f_{s}$ & $=$ & Sampling frequency & $f_{m}$ & $=$ & Nyquist frequency \\
\hline$S t_{D_{w}}=\frac{f D_{w}}{U_{\infty}}$ & $=$ & Strouhal number & $\Delta t$ & $=$ & Simulation time step \\
\hline$N_{i t}=2^{N}$ & $=$ & Number of samples & $\delta_{\omega}$ & $=$ & Vorticity thickness \\
\hline$\vec{\omega}$ & $=$ & Vorticity vector & $\Psi$ & $=$ & Fourier transformed wavelet function \\
\hline$\psi$ & $=$ & Wavelet in the time domain & $s, b$ & $=$ & Dilation and translation parameters \\
\hline$\gamma_{j, k}$ & $=$ & Wavelet coefficients & $T$ & $=$ & Threshold value \\
\hline$k$ & $=$ & Wavenumber & $\omega=2 \pi f$ & $=$ & Pulsation \\
\hline$\chi$ & $=$ & Goodness-of-fit test variable & $v_{\varphi}=k^{-1} \omega$ & $=$ & Phase velocity \\
\hline$\tau$ & $=$ & Correlation time-delay & $\tilde{\Delta}$ & $=$ & Dimensionless Laplace operator \\
\hline
\end{tabular}

\footnotetext{
*PhD student, DAAA - Dept. of Aerodynamics, Aeroelasticity and Acoustics, antoine.hajczak@onera.fr

${ }^{\dagger}$ Research scientist, DAAA - Dept. of Aerodynamics, Aeroelasticity and Acoustics, laurent.sanders@onera.fr

${ }^{\ddagger}$ Scientific advisor, DMPE - Dept. of Multi-Physics for Energetics, francois.vuillot@ onera.fr, AIAA senior member

${ }^{\S}$ Associate professor, Sorbonne Université - Institut Jean le Rond d'Alembert, philippe.druault@upmc.fr
} 


\section{Introduction}

INDING gear noise is now recognized as a major contributor to the total noise generated by a modern civilian aircraft. LThanks to the continued progress achieved in computational fluid dynamics, noise predictions of landing gear moderately complex geometries become affordable and enable deeper analyses of the noise generating processes. By reducing the problem of sound generation by a turbulent flow to a problem of linear acoustics with a distribution of equivalent sources, the acoustic analogy provides an attractive framework for landing gear noise predictions. On the other hand, this approach may not be the best suited to give detailed insight into the mechanisms involved in noise generation as the physical meaning of the equivalent source terms is not always straightforward. Yet, precise understanding of the physical mechanisms is of paramount importance as it can drive the design of low noise strategies (passive or active flow control for example). This understanding can be improved, for example, by studying the correlation between near-field pressure and relevant aerodynamic quantities.

In practice, an aeroacoustic problem involves two regions (the flow region and the far-field) with very different behavior, separated by a third region (the near-field), the latter being a grey zone that makes the continuous transition between the two former. In the flow region, pressure perturbations are essentially incompressible compensations of the local turbulent velocity fluctuations that result from the Biot-Savart law and are convected with the mean flow. Therefore, they are called hydrodynamic pressure, or pseudo-sound. On the other hand, in the linear far-field region, the pressure perturbations are exclusively due to the compressible nature of the propagation medium and travel at the speed of sound. They are then naturally called acoustic perturbations. The magnitude ratio between hydrodynamic and acoustic perturbations increases with decreasing Mach number. In the near-field, the pressure perturbations are then often assimilated to a combination of acoustic and hydrodynamic fluctuations. Due to computational resources limitations, compressible flow simulations are often restricted to the near-field of the flow considered. Hence, in CFD data, acoustic fluctuations can be somehow hidden in the high amplitude hydrodynamic fluctuations. Then, a filtering procedure can be necessary to extract the acoustic pressure, and enable further analysis of the acoustic field, for example with causality methods such as direct correlations, LSE, etc. This has been achieved by several authors with different mathematical tools. For instance, the so-called wavenumber-frequency filtering technique has been used with success by Tinney \& Jordan [1] and Kerhervé et al. [2] to isolate the acoustic component in the near-field of a turbulent jet. Similarly, the acoustic component has been extracted from the near field of a mixing layer by Druault [3] using a POD filtering strategy combined with the wavenumber frequency filtering method. Another filtering technique, introduced by Grizzi \& Camussi [4], uses a mathematical transformation alternative to the classical Fourier transform that has gained interest recently: the wavelet transform. The idea is that, since it is localized in both the physical and the frequency space [5], it is better suited for capturing coherent structures, as pointed out by Farge [6], and thus the hydrodynamic component related to them. Wavelet-based separation techniques have been further developed by Mancinelli et al. [7] who recently proposed three new procedures to filter the wavelet coefficients associated with hydrodynamic pressure. To the authors' knowledge, almost all the studies dedicated to near-field pressure wavelet filtering have focused on jet noise.

The aim of this paper is to assess the use of these new wavelet separation techniques in the near-field of the shear layer developing over the round cavity installed in a landing gear wheel taken from the simplified geometry LAGOON [8]. Besides the existent work of Mancinelli et al. [7], the originality of the results presented in this paper resides in three points. Firstly, the present study will make use of numerical data instead of experimental signals, hence presenting much shorter signals, with sampling frequencies potentially very high with respect to acoustic frequencies to meet low CFL number requirements. Secondly, these wavelet based separation methods have so far mostly been used in flow configurations where the Mach number was typically higher than 0.5. In the present work, the flow Mach number is set to 0.23 , so the ratio between acoustic and hydrodynamic disturbances is expected to be lower. Finally, in this analysis, the spatial decay of the filtered pressure field will be investigated by varying the distance between the flow and the microphone where the filtering methods are applied.

The paper is organized as follows: in a first section, the flow simulation and the shear layer development are recalled based on a previous study [9]. In a second section, the basics of the wavelet transform and the wavelet separation procedures are first briefly presented, before the implemented algorithms are used on near-field pressure data for one given microphone position. Finally, the ability of the wavelet-based methods to remove the pseudo-sound while retaining an acoustic behavior of the pressure field is assessed by studying the decay of the filtered acoustic pressure with distance. 


\section{Flow Simulation}

This section is a brief reminder of the LAGOON wheel cavity geometry and shear layer growth. More details about the computational setup, mesh description and mean and unsteady flow features can be found in de la Puente [9].

\section{A. Geometry description}

The LAGOON wheel has a diameter $D_{w}=3 \times 10^{-1} \mathrm{~m}$ and thickness $H_{w}=9 \times 10^{-2} \mathrm{~m}$. A shallow circular cavity of radius $R=0.27 D_{w}$ and depth to diameter ratio $\kappa=\frac{H}{2 R}=0.23$ is located in its inner part. A notable difference relatively to existing studies of circular cavity noise is the presence of the rounded tyre that forces the flow to detach at $\frac{x}{R}=-1.39$ due to an adverse pressure gradient before it can reach the cavity upstream edge. Cavity noise is known to be dependant on the incoming boundary layer profile [10], so this feature makes the specificity of the present case. In regard of this particular geometry, the depth to diameter ratio can be considered to lie within $0.17 \leq \kappa \leq 0.28$. In any case, as $\kappa \leq 1$, no strong tone is expected to take place [11]. To summarize, the main dimensions of the wheel cavity are presented in a median plane in figure 1 .

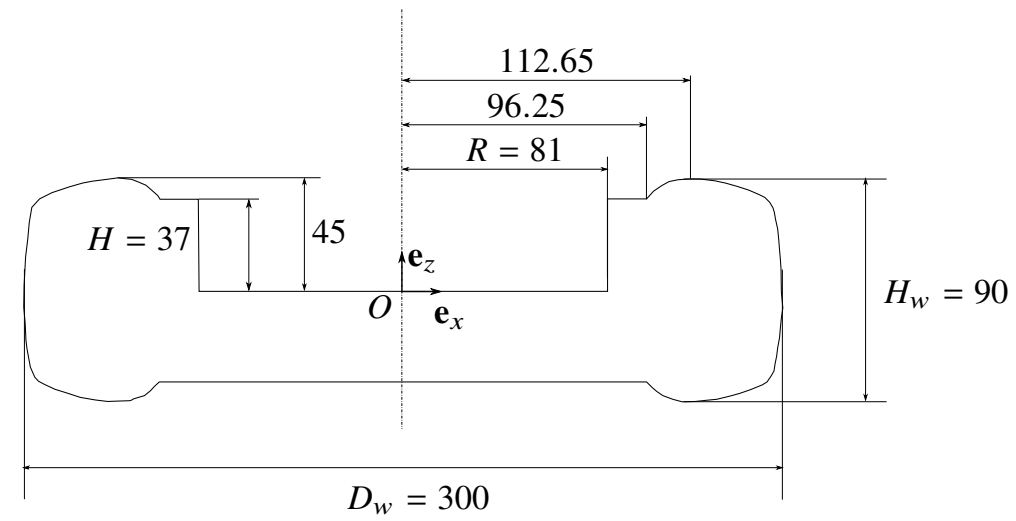

Fig. 1 Main dimensions (in mm) of the LAGOON wheel and cavity

\section{B. Mesh and computational setup}

ONERA's in-house code CEDRE is used in this study to solve the full compressible Navier-Stokes on a tetrahedral volume mesh with prism layers at the physical boundaries of the body with a Z-DES/SA turbulence model [9]. The flow Mach number is $M=\frac{U_{\infty}}{c_{0}}=0.23$ and directed in the $x$ direction such as $\vec{U}_{\infty}=U_{\infty} \vec{e}_{x}$. The prism layers are designed to obtain $y^{+} \sim 2$ at the walls, except on the cavity floor where a wall law is applied as the flow is expected to be mostly detached. Two concentric volume refinements are present over the cavity that allow a maximum frequency supported by the mesh $f_{C F D^{\sim}} 10 \mathrm{kHz}$ in the shear layer, or equivalently a Strouhal number based on the wheel diameter $S t_{D_{w}}=38$. The time step chosen for the simulation is $\Delta t=5 \times 10^{-6} \mathrm{~s}$. The near pressure field is stored at each simulation time step to obtain a total number of $N_{i t}=2^{16}$ iterations. As will be seen later on, a number of samples equal to a power of 2 is needed for the application of the discrete wavelet transform. The power spectral densities are computed with the Welch estimator by averaging Hann-windowed blocks to obtain a frequency resolution of about $50 \mathrm{~Hz}$.

\section{Flow features in the cavity median plane}

The mean streamwise velocity, along with several streamwise velocity profile is presented in figure 2a. The flow detachment is clearly visible at $x=-1.39 R$, while at the symmetric position on the opposite side of the wheel presents a short reattachment until $x=1.5 R$. Due to the circular shape of the cavity, the flow detachment is expected to take place at other locations for other angular position. However, as the turbulent structures over the cavity remain mostly bidimensional, the analysis is focused on the $(y=0)$ plane.

The growth of the shear layer has been characterized by de la Puente [9] in terms of vorticity and momentum thickness. In particular, as the hydrodynamic pressure is directly related to the vorticity, we recall here the initial vorticity thickness 
at the upstream cavity edge in the plane $(y=0)$ :

$$
\delta_{\omega_{0}}=\left.\frac{U_{\infty}}{\max _{z}\left(\frac{\partial u(x, y, z)}{\partial z}\right)}\right|_{\frac{x}{R}=-1}
$$

The vorticity thickness is a measure of the transverse dimension of a vortex. Its growth past the cavity is presented in figure $2 \mathrm{~b}$ In the present case, $\delta_{\omega_{0}}=0.13 H$, and the vorticity thickness growth follows a linear law between $\frac{x}{R}=-1$ and $\frac{x}{R}=0.1$. The growth then saturates with a still linear but much slower increase between $\frac{x}{R}=0.1$ and $\frac{x}{R}=1$.

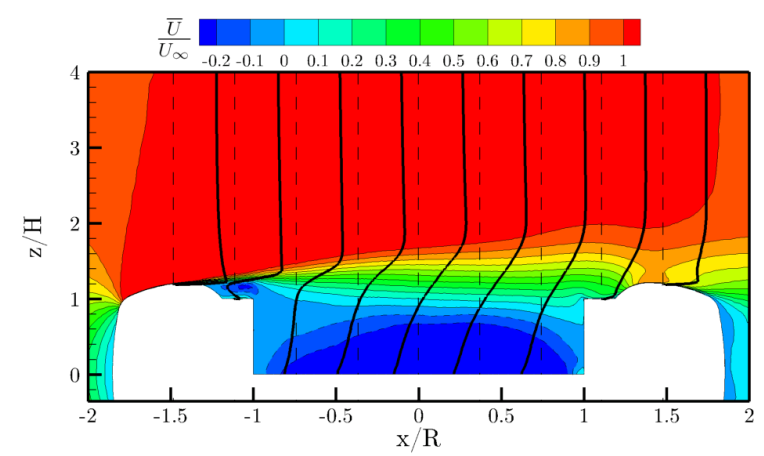

(a) Adimensioned mean streamwise velocity $\bar{U} / U_{\infty}$ in the wheel median plane and (-) velocity profiles

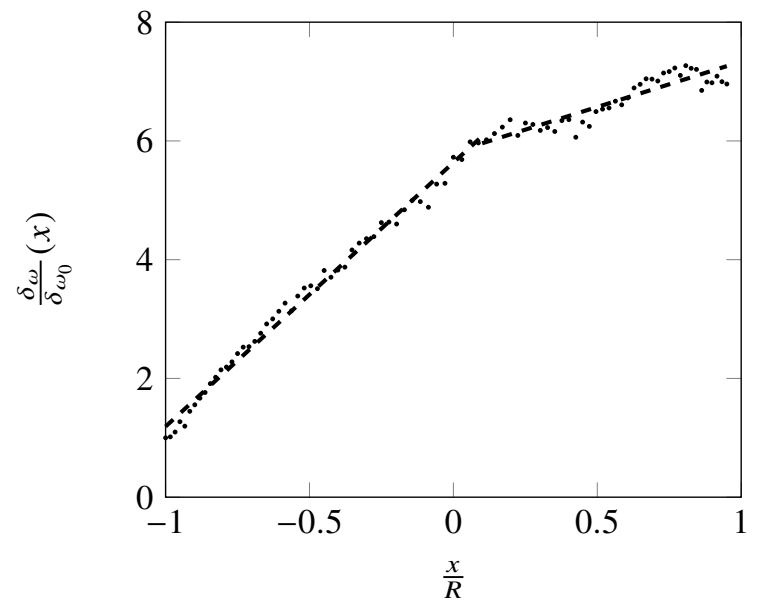

(b) Shear layer vorticity thickness $\delta_{\omega}(x)$ over the cavity adimensioned by the initial vorticity thickness at $\frac{x}{R}=-1$

\section{Fig. 2 Mean streamwise velocity and vorticity thickness evolution in the median plane over the cavity}

A snapshot of the instantaneous vorticity vector norm is depicted in figure 3. A vortex pairing is visible at the position where the vorticity thickness linear growth breaks, namely around $x / R=0.1$.

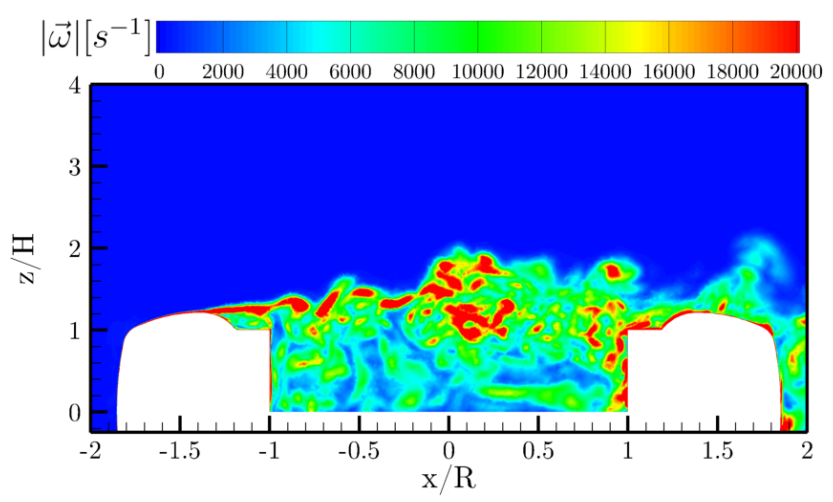

Fig. 3 Snapshot of the instantaneous vorticity norm $|\vec{\omega}|$ in the median plane of the wheel

\section{Near-field pressure filtering}

\section{A. The wavelet transform}

In this section, the mathematical definition of the wavelet transform will first be given, along with its most important properties. Then, the different existing filtering procedures will be recalled. The reader interested in the mathematical 
aspects of the wavelet transform will find a thorough overview in Mallat [5]. For the original articles in which the wavelet based separation procedures were proposed, please refer to Grizzi \& Camussi [4] and Mancinelli et al. [7].

\section{Mathematical definition}

A wavelet $\psi$ is a function that satisfies the so-called admissibility condition:

$$
\int_{-\infty}^{+\infty} \frac{|\Psi(\omega)|^{2}}{|\omega|} d \omega=0
$$

Where $\Psi$ is the Fourier transform of $\psi$. The admissibility condition directly implies that $\Psi$ must vanish at zero-frequency, and therefore that $\psi$ has a band-pass spectrum. The continuous wavelet transform of a function $g$ is the projection of $g$ on the basis of the scaled and translated wavelets defined by:

$$
\left(\psi_{s, b}: t \longmapsto \frac{1}{\sqrt{s}} \psi\left(\frac{t-b}{s}\right)\right)_{(s, b) \in \mathbf{R}^{2}}
$$

Thus, the wavelet coefficients are defined by:

$$
\gamma_{s, b}=\left(g \mid \psi_{s, b}\right)=\int g(t) \psi_{s, b}^{*}(t) d t
$$

The discrete counterpart of this continuous transform can be obtained by using a dyadic scale range for $s$. After posing $s=2^{-j}$ and $b=k s$, we get:

$$
\psi_{j, k}: t \longmapsto 2^{\frac{j}{2}} \psi\left(2^{j} t-k\right)
$$

and the discrete wavelet coefficients $\gamma_{j, k}$ obtained by convolution with the discrete time signal are such that:

$$
g(t)=\sum_{(j, k) \in \mathbf{Z}^{2}} \gamma_{j, k} \psi_{j, k}(t)
$$

The dyadic scale range ensure orthogonality of the wavelet transform, so the Parseval relation holds for the wavelet coefficients:

$$
\int|g(t)|^{2} d t=\sum_{(j, k) \in \mathbf{Z}^{2}}\left|\gamma_{j, k}\right|^{2}
$$

and the original signal can be reconstructed in the time domain with no loss of information knowing the wavelet coefficients.

As scaling in the time domain results in an inverse scaling in the frequency domain, it is actually possible to show that each scaled wavelet behaves like a band-pass filter covering a frequency interval corresponding to the considered scale. Each wavelet daughter has then a halved bandwidth as its support is dilated by a factor two as schematically illustrated in figure 4 If $a=\left[a_{0}, a_{1}, \ldots, a_{N_{i t}}\right]$ is a time signal of length $N_{i t}=2^{N}$ with a sampling frequency $f_{s}=2 f_{m}$, then the Fast Wavelet Transform algorithm returns a set of coefficients having the same number of elements ordered by slices of size $2^{j}$ with $0 \leq j \leq N-1$, the first coefficient corresponding to the mean $(f=0)$ value of the signal. For a given $j$, the $2^{j}$ corresponding coefficients $\gamma_{j}, \bullet$ contain the information in the frequency interval $\left[\frac{f_{m}}{2^{N-j}}, \frac{f_{m}}{2^{N-j-1}}\right]$ as follows:

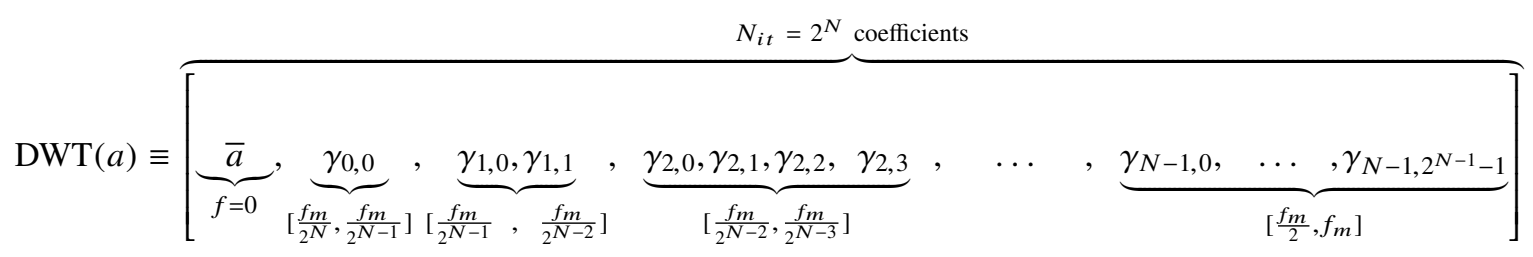

To summarize, the most interesting property of wavelets is that, as a consequence of eq. (1), they are localized both in the physical and the Fourier space. They are then expected to be better suited for capturing isolated events like the disturbance induced by a vortex than the classical Fourier transform, which has basis sine functions of infinite support in the physical space. As the wavelet coefficients are merely another way of representing a time signal, it is possible to 


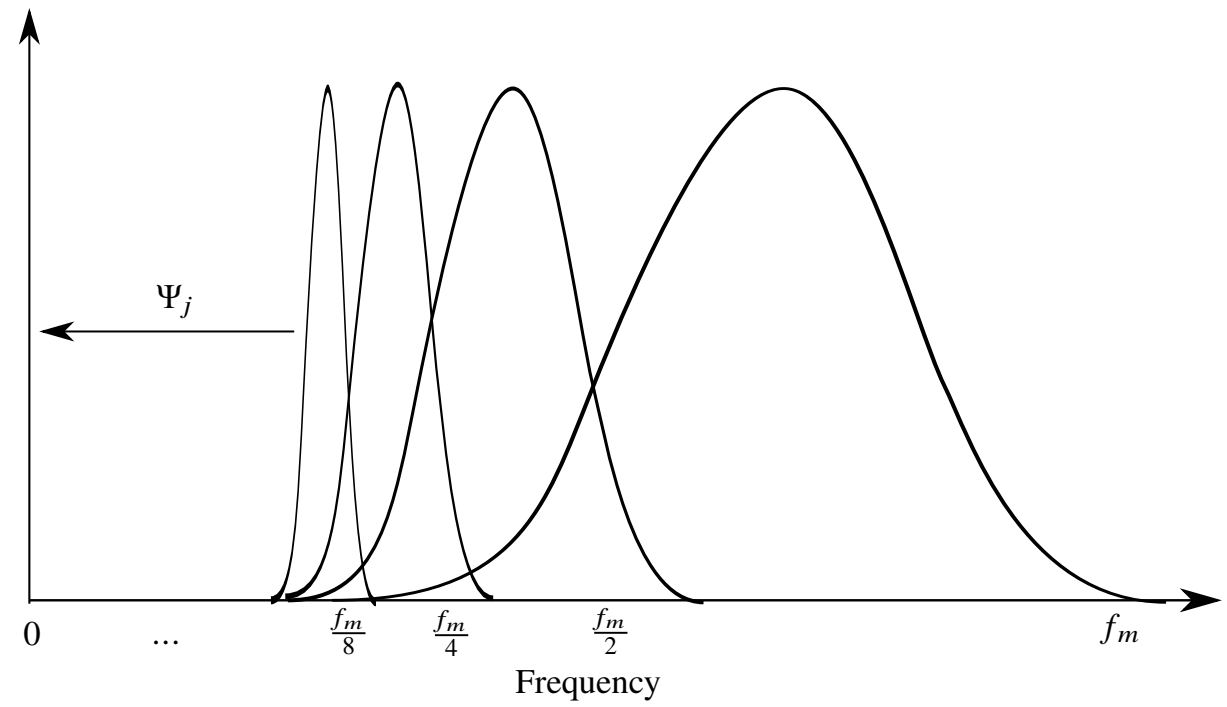

Fig. 4 Schematic representation of the scaled wavelets spectra in the Fourier space. As a result of the dyadic scaling, the bandwidth is halved and moved to lower frequencies as the wavelet support is dilated by a factor 2.

extract some features of this signal by resorting to a filtering procedure. All the existing filtering procedures consist in zeroing all the wavelet coefficients that exceed a threshold $T$ "conveniently" defined:

$$
\left(\gamma_{j, k}\right)_{<}=\left\{\begin{array}{ccc}
\gamma_{j, k} & \text { if } & \left|\gamma_{j, k}\right| \leq T \\
0 & \text { otherwise }
\end{array}\right.
$$

and then using the reconstruction formula (3) to recover the filtered signal in the time domain.

In practice, the determination of $T$ is performed thanks to an algorithm based on a physical feature of the expected acoustic component. The algorithms used in this paper are briefly described in the next paragraph.

\section{Existing filtering methods}

2.a. A Fourier-based method: the $k-\omega$ filter

When a spatio-temporal description of the near-field is available, for instance with a linear array of microphone in an experimental setup, or with numerical data obtained with a CFD solver, it is possible to extract the propagating component of the near field from the total pressure field by performing a two-dimensional Fourier transform both in space and time, giving the wavenumber frequency spectrum:

$$
\hat{p}(k, \omega)=\iint p(x, t) e^{i(k x-\omega t)} d t d x
$$

The propagating component, as opposed to the convective component, has to travel at an apparent speed that is at least $c_{0}-U_{\infty}$, and must therefore verify $|\omega / k| \geq c_{0}-U_{\infty}$. Acoustic waves can present an inclination angle that makes their apparent speed relatively to the array larger than the speed of sound. The acoustic component is then obtained by applying the following filter in the Fourier space:

$$
\hat{p_{a}}(k, \omega)=\left\{\begin{array}{ccc}
\hat{p}(k, \omega) & \text { if } & \left|\frac{\omega}{k}\right| \geq c_{0}-U_{\infty} \\
0 & \text { otherwise }
\end{array}\right.
$$

and then by performing an inverse Fourier transform to the filtered component back in the physical space:

$$
p_{a}(x, t)=\iint \hat{p_{a}}(k, \omega) e^{-i(k x-\omega t)} d k d \omega
$$


As pointed out by Mancinelli et al. [7], a drawback of this method is that, when applied to experimental data, the wavenumber resolution of the microphone $\Delta k$ is a direct limitation to the filtering at low $\omega$ values. Long arrays are then needed to get a better filtering of the low frequency component of the signal. While numerical simulations offer flexibility in the design of the virtual array, this can become problematic in experimental setups. To overcome this difficulty, they proposed three filtering procedures $W T 1, W T 2$ and WT3 in addition to the original method WT4 proposed by Grizzi \& Camussi [4].

\section{2.b. Wavelet-based methods}

- The WT1 method relies on the two-point correlation between the microphone where the filtering is performed and a reference signal in the acoustic far-field. Starting from an initial threshold value, the cross correlation between the filtered part and the far-field signal is computed until it reaches a maximum.

- The WT2 method is based on the assumption that the acoustic component of the pressure signal has a probability density function that follows a Gaussian distribution. This method then only requires the pressure signal at the point where the filtering is desired. Starting from an initial threshold, the signal is filtered and at each iteration compared with a reference Gaussian distribution through a $\chi^{2}$ test until a prescribed degree of similarity is found between the reference Gaussian distribution and the actual filtered signal probability density function.

- The WT3 is an existing method called Coherent Vortex Extraction (CVE) proposed by Rupper-Felsot et al. [12] that aims at extracting coherent structures. This methodology consists in a recursive filtering of the vorticity field based on the assumption that coherent structures are what remains after removing its Gaussian component. In the case of jet noise, Mancinelli et al. [7] used this method in a similar way, considering that the hydrodynamic pressure is directly related to the vorticity: the pressure signal is recursively filtered with a threshold based on the Donoho-Johnstone [13] denoising theorem. The algorithm stops when the number of wavelet coefficients associated with the incoherent part of the signal does not change from one iteration to the next. The signal associated with these coefficients is then transformed back to the physical space and is assimilated to the acoustic pressure.

- Finally, the WT4 method, introduced by Grizzi \& Camussi [4] does not require the knowledge of the far-field pressure, but another near-field probe located close to the point where the filtering is to be performed. Both time signals $s_{1}$ and $s_{2}$ are iteratively filtered into two components $s_{1}=s_{1 h}+s_{1 a}$ and $s_{2}=s_{2 h}+s_{2 a}$ where the subscript $h$ stands for the hydrodynamic component composed of the coefficients $\left(\gamma_{j, k}\right)_{>}$such that $\left(\gamma_{j, k}\right)_{>} \geq T$ and $a$ for the acoustic component represented by the remaining coefficients inferior to the threshold. The algorithm stops whenever:

- The cross-correlation peak $R_{s_{1 h}-s_{2 h}}$ corresponds to a convection velocity inferior to the mean velocity,

- The cross-correlation peak $R_{S_{1 a}-s_{2 a}}$ corresponds to a propagation velocity superior or equal to the apparent speed of sound $c_{0}-U_{\infty}$,

- The ratio between the first and second peak of the cross-correlation $R_{s_{1 h}-s_{2 h}}$ must be superior to a convenient value representative of the signal to noise ratio.

Other filtering methods based on the so-called Local Intermittency Measure of the wavelet scalogram (wavelet spectrum obtained with the continuous wavelet transform) have been used by other authors (see for example Grassucci et al. [14], or more recently Pérez Arroyo et al. [15]) but have not been tested in this work, although their use might constitute an interesting perspective.

\section{B. Application to the cavity shear layer at a single point}

The filtering procedures previously described have been implemented using the open-source wavelet library WaveLab 850 developed at Stanford University. In all cases, a Daubechies wavelet with 8 vanishing moments is used to perform the DWT of the signal. The results are expected to remain unaffected by the choice of the mother wavelet as reported in previous investigations (see for instance Kœnig et al. [16] or Camussi et al. [17]). The unsteady near-pressure field has been recorded on a linear array of microphone located at a vertical distance $r=13 \delta_{\omega_{0}}$ from the cavity edge as depicted in figure $5 \mathrm{a}$. It consists of 101 microphones equally spaced with a distance $\Delta x=\frac{D_{w}}{60}$, giving a wavenumber resolution $\Delta k=6.22 \mathrm{~m}^{-1}$. The microphone retained for the assessment of the filtering techniques is depicted by a red dot in figure $5 \mathrm{a}$ and is located at $x=0.62 \mathrm{R}$ and $z=2.7 \mathrm{H}$. A sample of the time signal at this location is presented in figure $5 \mathrm{~b}$, showing clearly the shear layer strong pressure perturbations directly related to a convected turbulent eddy. Here, such an intense event is visible at $\tau^{*}=23.9$ convective times based on the wheel diameter. 


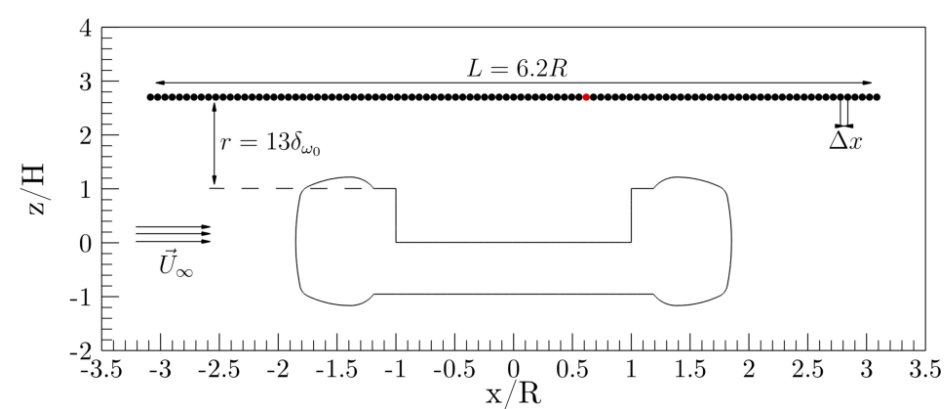

(a) Position of the reference point (red dot) for the assessment of the wavelet based separation techniques

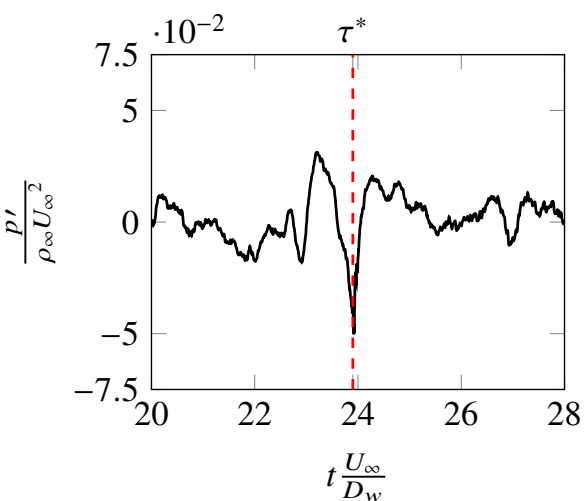

(b) Instantaneous normalized pressure time signal for an observer located at $\frac{x}{R}=0.62$ and $\frac{z}{H}=2.7$

\section{Fig. 5 Position of the reference point and sample of the time signal}

\section{1. $k-\omega$ filtering}

The wavenumber-frequency spectrum obtained on the linear microphone array has been filtered according to eq. 7. The unfiltered spectrum is presented in figure 6a with black lines corresponding to different apparent propagation speeds $v_{\varphi}=\omega k_{x}{ }^{-1}$. From now on, the original CFD pressure will be simply denoted $p^{\prime}$. Conversely, the component presumably assimilated to hydrodynamic pressure will be denoted $\left(p^{\prime}\right)_{>}$and the component assimilated to acoustic pressure $\left(p^{\prime}\right)_{<}$. Two contributions clearly appear with distinct propagation speeds. The hydrodynamic component indicates a convection velocity of about $U_{c}=0.77 U_{\infty}$, while the acoustic component is characterized by a velocity superior to $c_{0}-U_{\infty}$. The sign of the velocity is also visible thanks to the slope of the two lobes, and clearly highlights the upstream propagation associated with cavity noise, characterized by negative values of $k_{x} D_{w}$ and positive $S t_{D_{w}}$. After reconstruction in the physical space, the time signal and spectra of the different contributions is presented in figures $6 \mathrm{~b}$ and $6 \mathrm{c}$. The intense event occurring at $\tau^{*}$ is not present in the acoustic component, indicating that the $k-\omega$ filter is able to remove with success the biggest hydrodynamic events.

\section{WT1 - Correlation with a far-field observer}

A pressure signal in the far-field of the wheel has been obtained thanks to a FW-H computation, with the integration surface taken coincident with the physical surface of the wheel. The position of the far-field observer is $\left(\frac{x}{D_{w}}, \frac{y}{D_{w}}, \frac{z}{D_{w}}\right)=$ $(-2.5,0,4.33)$. According to the procedure WT1, the CFD signal obtained in the near-field of the wheel shear layer has been iteratively filtered by thresholding its wavelet coefficients until the cross-correlation between the filtered part and the far-field signal reached a maximum. The optimal cross-correlation is plotted in figure $7 \mathrm{a}$ and illustrates how removing the biggest wavelet coefficients has increased the correlation with the "pure" acoustic signal in the far-field. Moreover, the time delay $\tau$ corresponding to the correlation peak is such that $\tau c_{0} / D_{w}=5.45$, which is consistent with the upstream propagation between the CFD point and the FW-H microphone separated by a distance equal to $5 D_{w}$. The resulting filtered time signals and spectra are reported in figures $7 \mathrm{~b}$ and $7 \mathrm{c}$. As expected, the hydrodynamic component is dominant in the low frequency range. Finally, it should be stressed that the intense aerodynamic event at $\tau^{*}$ has been successfully comprised in the hydrodynamic part of the signal, thus illustrating the capacity of the wavelet transform to capture localized events.

\section{WT2 - Similarity of the probability density function to a Gaussian distribution}

The broadband noise radiated by the wheel in the far-field is expected to follow a Gaussian distribution. Accordingly, the near-field acoustic component that propagates to the far-field is supposed to have a probability density function that fits a Gaussian reference distribution. The threshold has been varied from its highest possible value $T_{m}=\max \left(\left|\gamma_{j, k}\right|\right)$ until a $\chi^{2}$ goodness of fit test was verified. The pdf were computed using 51 bins, and the tolerance value for the assessment of the test was set to $\epsilon=10^{-4}$ as proposed by Mancinelli et al. [7]. The probability density function of the original and filtered pressure are shown in figure $8 \mathrm{a}$ where the standard Gaussian distribution is also presented 


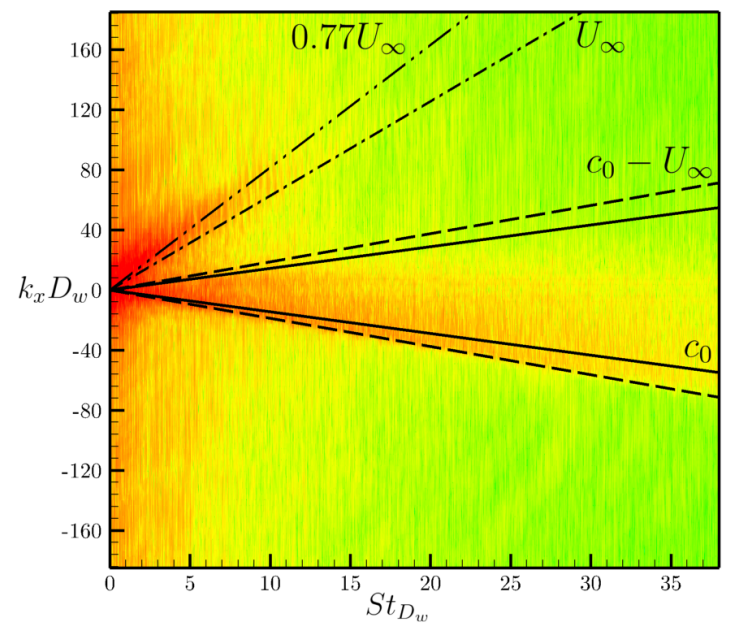

(a) Wavenumber-frequency spectrum of the pressure stored along the linear array. Solid: $\omega k_{x}{ }^{-1}=c_{0}$, dashed: $\omega k_{x}{ }^{-1}=c_{0}-U_{\infty}$, dash-dot: $\omega k_{x}{ }^{-1}=$ $U_{\infty}$, dash-dot-dot: $\omega k_{x}{ }^{-1}=0.77 U_{\infty}$

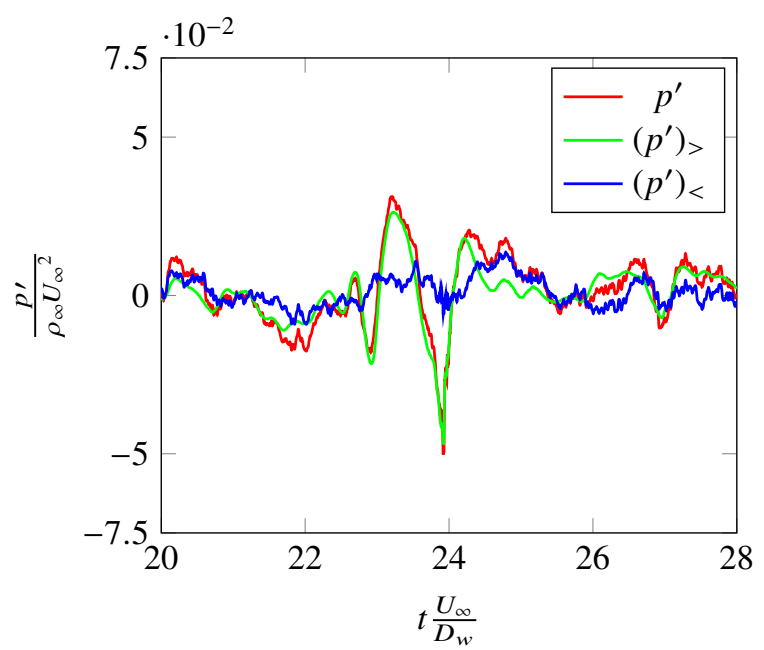

(b) Superimpositon of the reference signal onto the filtered components obtained with the $k-\omega$ procedure

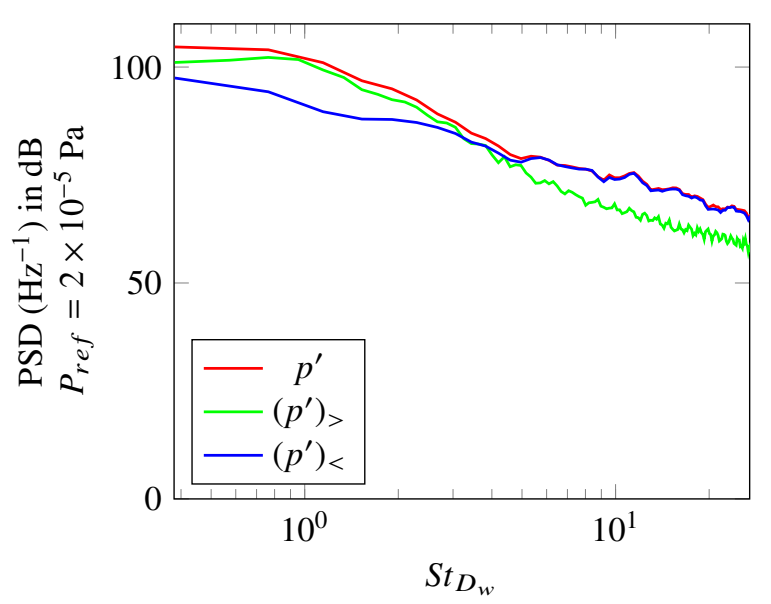

(c) PSD of the total and extracted components

Fig. 6 Results of the $k-\omega$ filtering procedure with the filter set to extract the component propagating at $c_{0}-U_{\infty}$

with black dots. The original pressure exhibits the typical "tails" for extreme values of $p^{\prime}$ that are associated with rare high-amplitude perturbations caused by the passage of a vortex. After the filtering procedure, the agreement with the reference distribution shows that the value chosen for $\epsilon$ allows a good removal of the non-Gaussian part of the signal. The time signals obtained after the $W T 2$ procedure are presented in figure $8 \mathrm{~b}$ along with the spectra in figure $8 \mathrm{c}$ The spectrum of the filtered component seems pretty similar to the one obtained with the WT1 method in figure $7 \mathrm{c}$ A synthetic comparison between the methods will be presented in the end of the section.

\section{WT3 - Recursive filtering based on the Coherent Vortex Extraction procedure}

The WT3 algorithm only needs the pressure in the near-field. It is based on the assumption that the hydrodynamic component is the "coherent" part of the signal, namely what remains after the Gaussian "incoherent" part has been removed from the signal, in accordance with a definition of Ruppert-Felsot [12] for the eduction of coherent structures. Using a threshold value based on the theorem of Donoho \& Johnstone [13], the part of the signal represented by the 


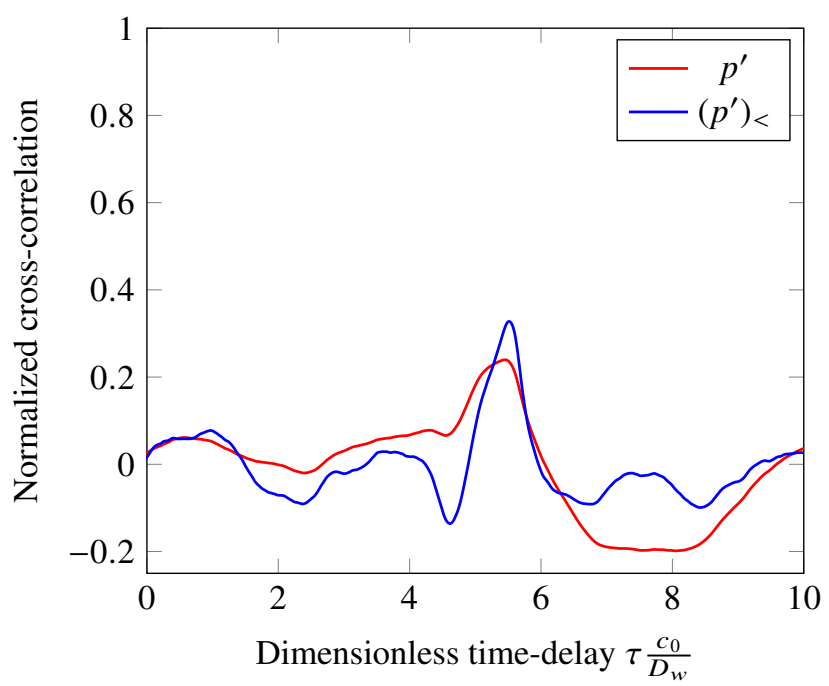

(a) Cross-correlation between (-) far-field and original signal, and (-) far-field and filtered signal

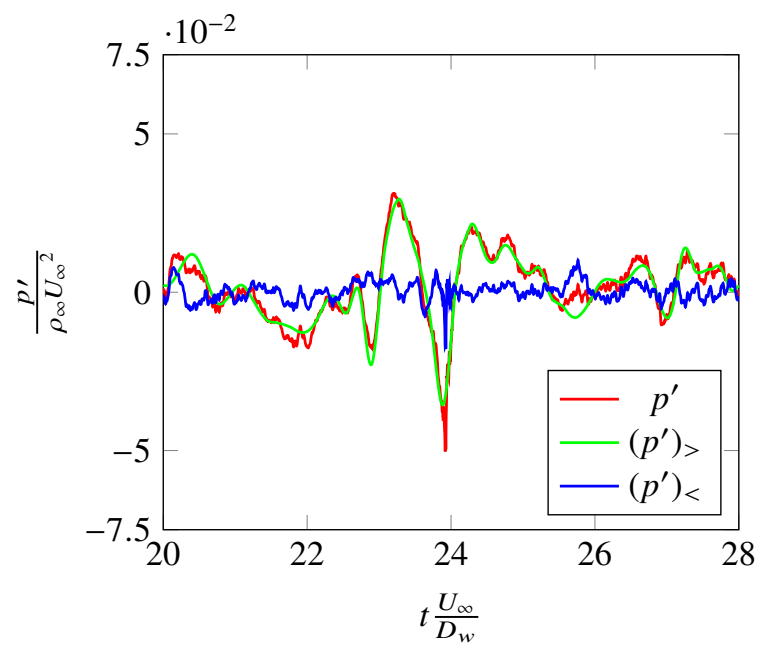

(b) Time signals after the WT1 filtering procedure

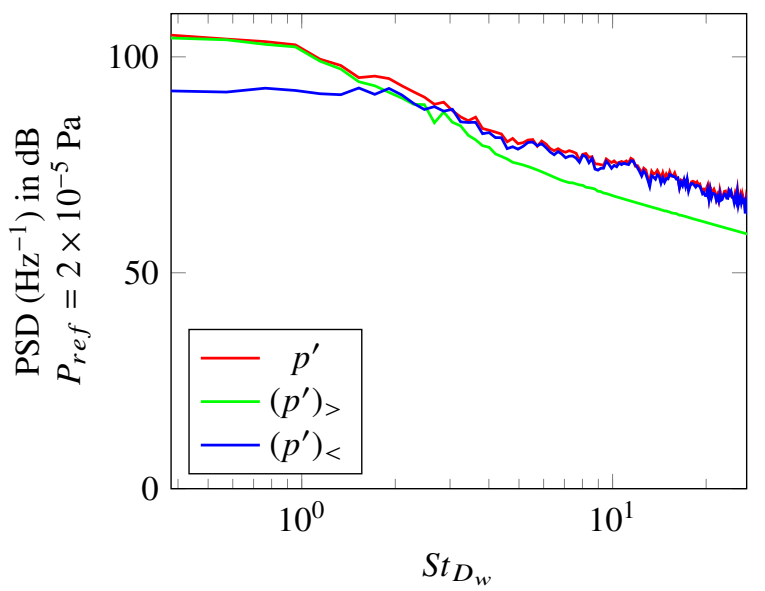

(c) PSD of the original signal and filtered components

Fig. 7 Results obtained with the WT1 procedure

coefficients inferior to the threshold is recursively filtered by adapting the threshold value at each iteration. The value of the threshold, as well as the number of coefficients representing the incoherent part is depicted in figure 9a for each iteration until convergence is reached. In the present case, the convergence of the algorithm requires 32 iterations to be reached. The main idea behind this procedure is that the hydrodynamic perturbations have a sparse representation on the wavelet basis, meaning that very few coefficients are necessary to represent it. Here, the compression ratio (number of coefficients representing the incoherent part divided by the total number of coefficients) is about $76 \%$, which is quite low compared to values found in the literature (e.g. [7], [12]). The algorithm convergence seems too slow, as the algorithm seems to somehow over-filters the signal by removing too many coefficients which is further confirmed by the spectra of the different contributions presented in figure $9 \mathrm{c}$ The filtered component thus obtained is Gaussian but clearly not associated with a physical acoustic component of the pressure field. In fact, recalling the Nyquist frequency $f_{m}=\frac{1}{2 \Delta t_{C F D}}=100 \mathrm{kHz}$ and the link between the wavelet coefficients and the bandpass filter behavior of the scaled wavelets, it appears that a large number of coefficients represents the random background noise fluctuations of the simulation. In the present case, the mesh cutoff frequency is about $10 \mathrm{kHz}$. Hence, all coefficients $\gamma_{j, k}$ with $j \geq 13$ are representative of the signal at frequencies between $12.5 \mathrm{kHz}$ and $100 \mathrm{kHz}$, which is well above the CFD cutoff. In order 


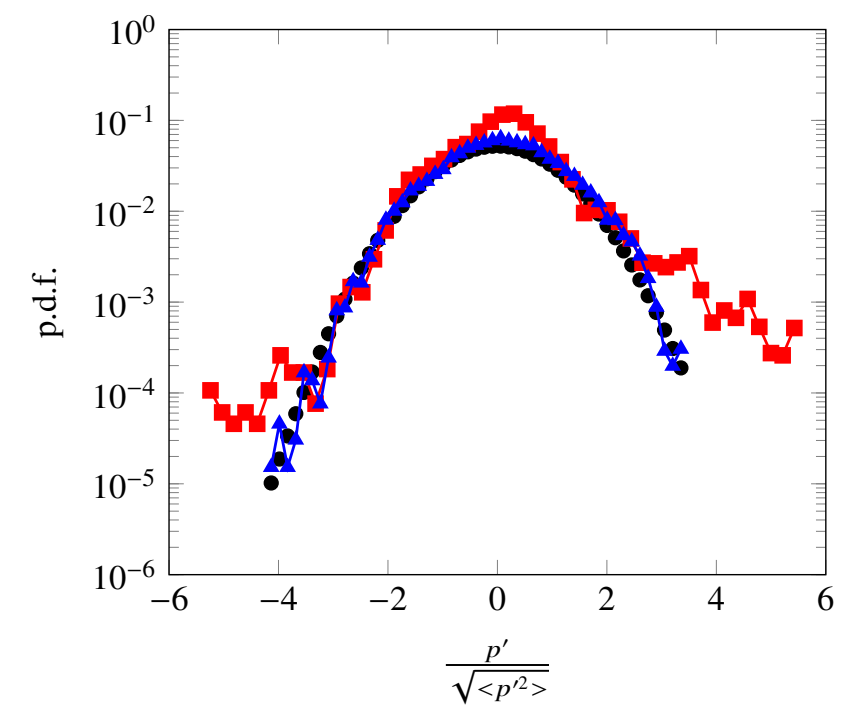

(a) Probability density function of the reference signal ( $\square$ ) and the signal filtered with a $\chi^{2}$ goodness-of-fit criterion ( $\mathbf{\Delta}$ ) with respect to a standard Gaussian distribution $(\bullet)$

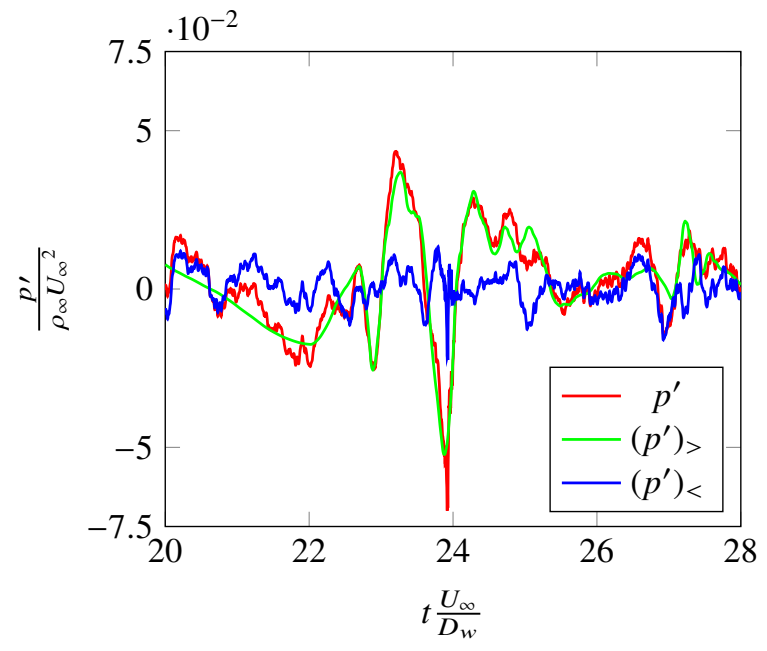

(b) Time signals after the WT2 filtering procedure

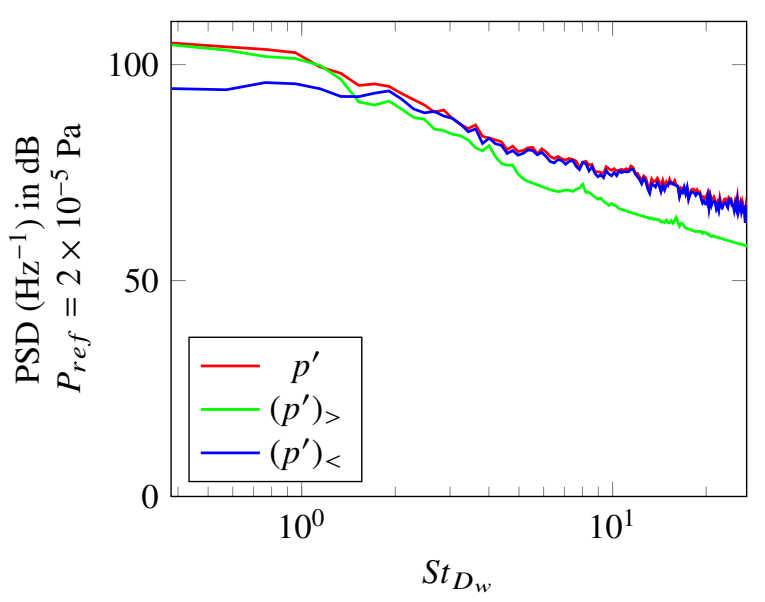

(c) PSD of the original signal and filtered components

Fig. 8 Results obtained with the WT2 procedure

to overcome this problem, the signal has been resampled to a sampling frequency close to the mesh cut-off frequency of the simulation $f_{C F D}=10 \mathrm{kHz}$. Doing so, one ensures that the wavelet coefficients at all scales are physical. In order to avoid aliasing, the signal has been Fourier transformed and the Fourier coefficients above $10 \mathrm{kHz}$ have been set to zero before performing the inverse transform back in the physical space. The procedure has then been applied to the resampled data giving the results presented in figure 10 . Only 11 iterations are now needed to reach convergence. The final threshold value is about 100 times higher than the one obtained when the signal was not decimated, resulting in a filtered signal that presents a rather flat low frequency spectrum and coincident with the original signal for $S t_{D_{w}} \geq 5$. The compression ratio has increased to a value of $91 \%$, in better accordance with the assumption that the hydrodynamic component has a sparse representation on a wavelet basis.

\section{WT4 - Cross-correlation between two near-field observers}

Finally, the original procedure proposed by Grizzi \& Camussi [4] has been assessed by considering the same pressure probe at $(x=0.62 R, z=2.7 \mathrm{H})$ and another near-field point located downstream at a distance $\Delta x$ such that 


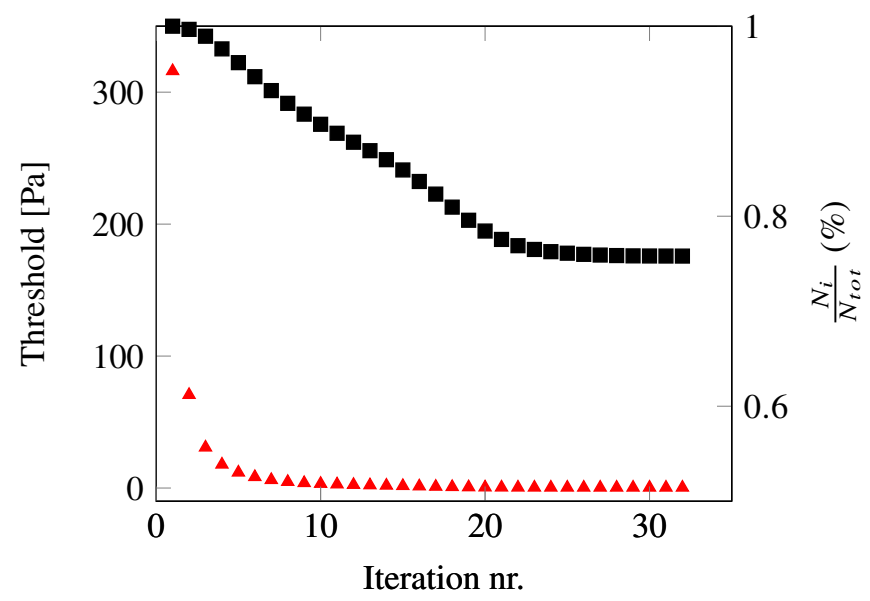

(a) Threshold value $(\boldsymbol{\Delta})$ and proportion of incoherent coefficients $\left(\gamma_{j k}\right)_{<}$

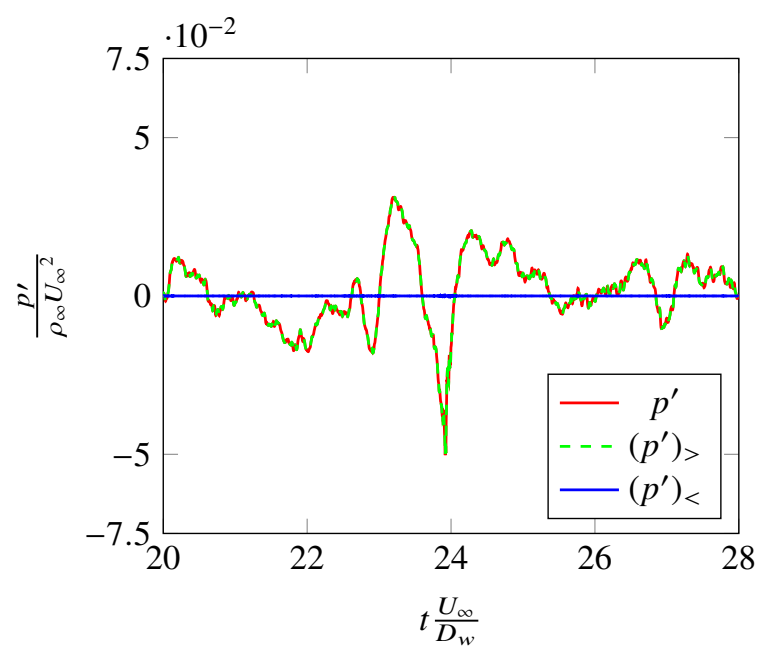

(b) Time signals after the WT3 procedure

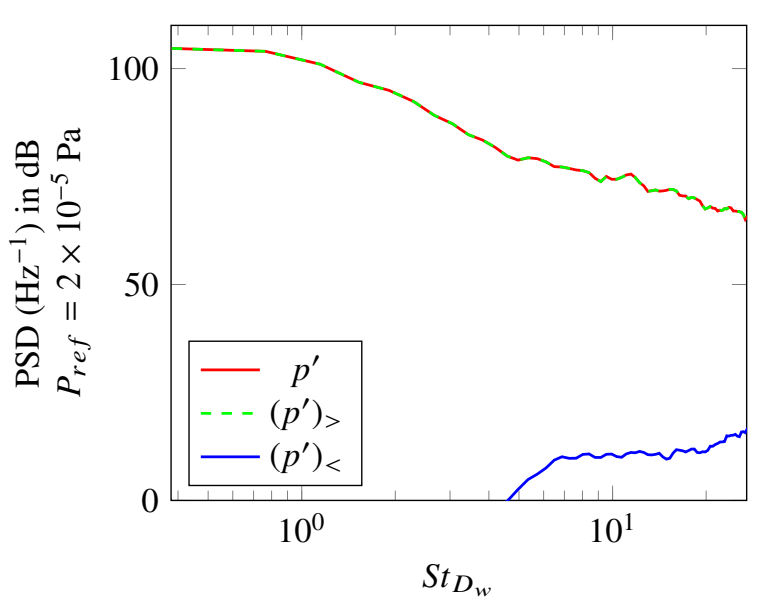

(c) PSD of the original signal and filtered components

Fig. 9 Results obtained with the WT3 procedure : $N=16$ and $f_{m}=100 \mathrm{kHz}$

$\frac{D_{w}}{\Delta x}=30$. A slight modification of the original algorithm was made on the third criterion. Instead of comparing the ratio between the first two peaks of $R_{s_{1 h}-s_{2 h}}$ to a user-defined value, it was found more robust to maximize the cross-correlation $R_{S_{1 a}-s_{2 a}}$ between the acoustic components. Doing so, the algorithm does not require any adjustment from one dataset to another, while maintaining the original physical ideas underlying the filtering process.

The two signals are then filtered according to this modified algorithm procedure until the convergence criteria are verified. The cross-correlation coefficient between the hydrodynamic and acoustic parts of both microphones is displayed after convergence in figure $11 \mathrm{a}$. The results are coherent with the expected physics : the time delays corresponding to the maxima are in accordance with the velocity for both types of perturbations: $\tau_{a} c_{0} / \Delta x=-1$ and $\tau_{h} c_{0} / \Delta x=4.6 \simeq 1 / M$. The convection velocity associated with this time delay is about $U_{c}=0.95 U_{\infty}$, which is different from the value obtained with the $k-\omega$ analysis. This difference could be attributed to the local character of the wavelet procedure, while the $k-\omega$ analysis is based on an extended spatial zone. Furthermore, the negative sign of the time delay associated with the acoustic part shows the upstream propagation of the circular cavity noise. The spectra of the signal components are presented in figure $11 \mathrm{c}$ and, like in figure $7 \mathrm{c}$ indicate that pseudo sound in concentrated in the low frequency range. It should be noted that, as opposed to the $k-\omega$ filter and both WT1 and WT2 methods, but similarly to WT3, the low frequency part of the spectrum presents way lower levels. 


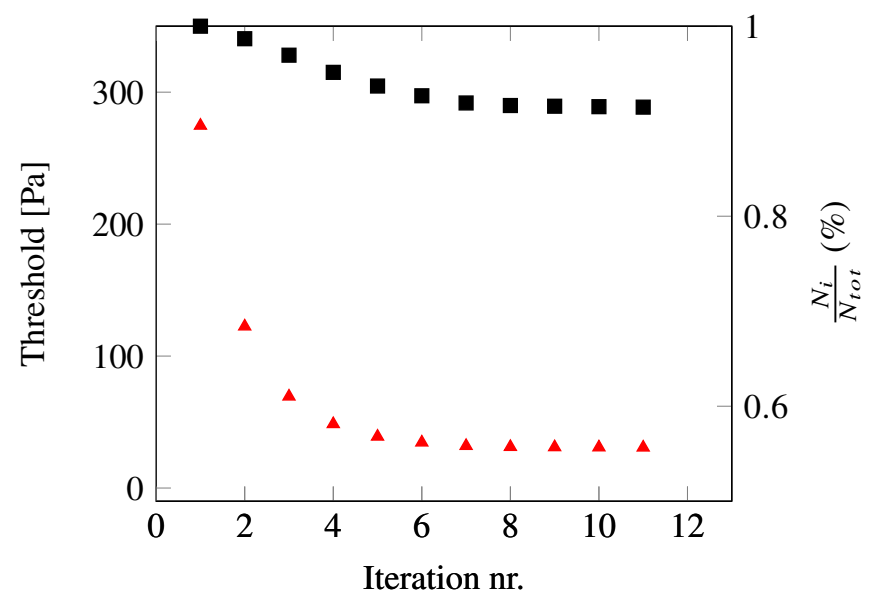

(a) Threshold value $(\boldsymbol{\Delta})$ and proportion of incoherent coefficients $\left(\gamma_{j k}\right)_{<}$

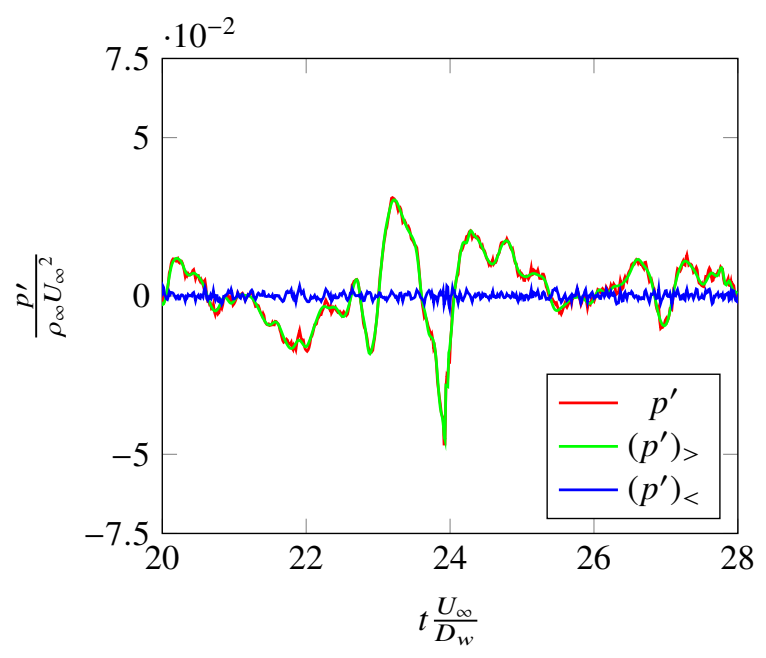

(b) Time signals after the WT3 procedure

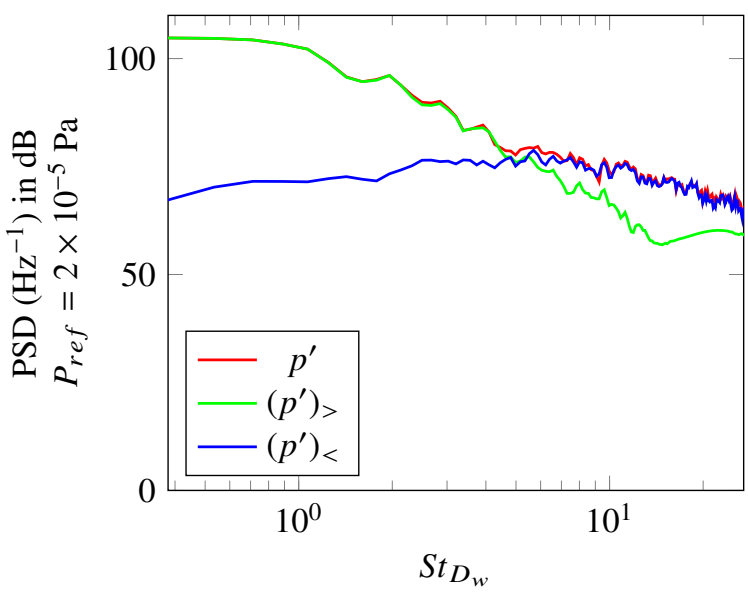

(c) PSD of the original signal and filtered components

Fig. 10 Results obtained with the WT3 procedure : $N=12$ and $f_{m}=10 \mathrm{kHz}$

\section{Comparison of the methods}

In order to compare the four methods, the spectra of the filtered part is plotted in figure 12 for the four methods. The spectrum corresponding of the original signal, as well as the result of the $k-\omega$ method have also been plotted for comparison. Among the tested methods, two types of spectra emerge from the results. On one hand, the WT1, $W T 2$ and $k-\omega$ methods that still present a low frequency hump but in a lesser extent than the original signal, thus indicating that some energy associated with the low frequency hydrodynamic component has been removed. On the other hand, the WT3 and WT4 method present spectra that differ by several decades in the low frequency range and that, interestingly, exhibit a shape in the low frequency range that looks closer to the results obtained by Mancinelli et al. [7] who had a rather flat spectrum in the low frequency range.

While WT1 and WT4 rely on two-points cross-correlations, WT2 and WT3 rely on the assumption that the acoustic component of the near-field has a Gaussian pdf. The pdf of the extracted component is then plotted for these two methods in figure 13 for comparison. With both methods, the extracted component follows the standard Gaussian distribution, confirming that convergence is reached in both cases. On the other hand, the important discrepancies between the levels predicted with both methods seems to evidence that the Gaussian assumption alone is not sufficient to completely characterize the acoustic component of the pressure field. 


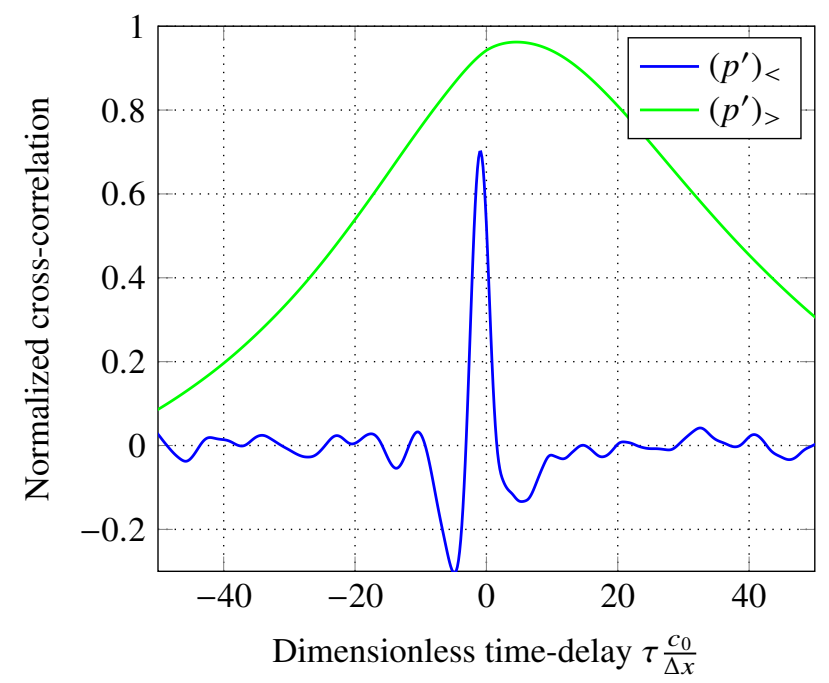

(a) Cross-correlation between the separated components of two microphones separated by a distance $\Delta x=\frac{D_{w}}{30} .(-): R_{s_{1 a}-s_{2 a}} ;(-): R_{s_{1 h}-s_{2 h}}$

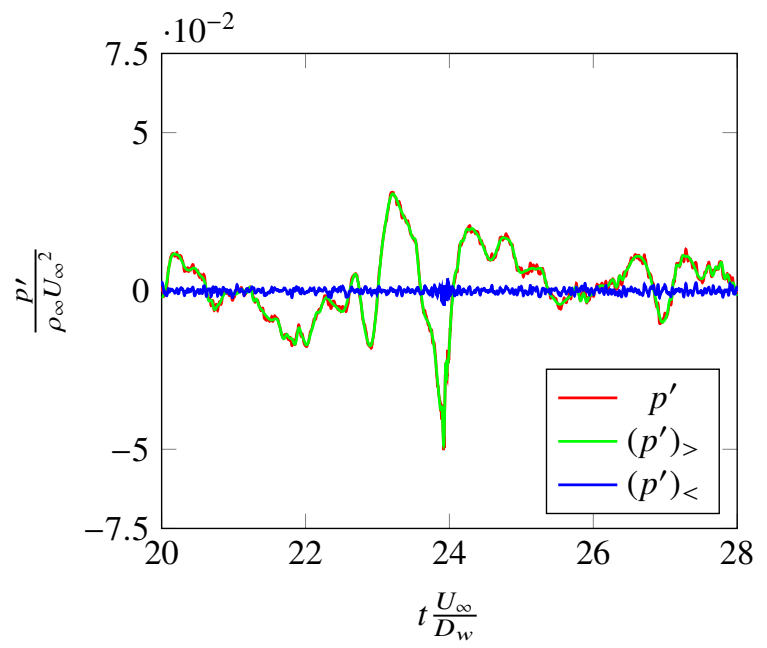

(b) Time signals after the WT4 procedure

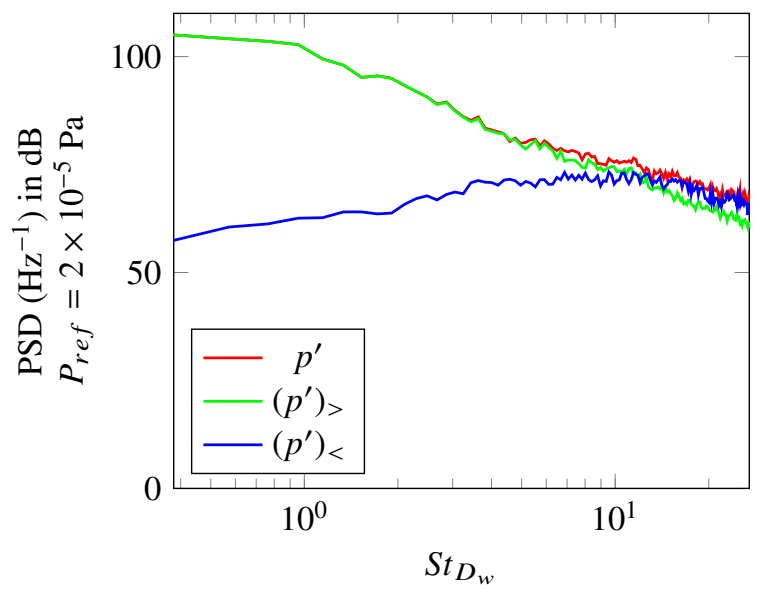

(c) PSD of the original signal and filtered components

Fig. 11 Results obtained with the WT4 procedure

\section{Application to near and far-field microphones - spatial decay of the pressure field on a vertical line}

For a given observer distance $r$, an approximate definition of the acoustic near and far-field can be given based on the product $k_{0} r$, where $k_{0}$ is the acoustic wavenumber under consideration. Following a dimensional analysis (see for example Crighton [18]), one can show that the equation governing the pressure field becomes a Poisson equation in the low wavenumber limit $k_{0} \rightarrow 0$. Indeed, if $L$ is a characteristic length of the pressure field and $\tilde{\Delta}$ the adimensioned Laplace operator:

$$
\left(\tilde{\Delta}-\left(k_{0} L\right)^{2}\right) p \simeq \tilde{\Delta} p \text { when } k_{0} L<<1
$$

Conversely, an observer located at a distance $r$ from the sources can be considered in the acoustic propagating field as soon as $k_{0} r \geq 1$. In the present case, a reasonable gross estimate of the source-observer distance could be the vertical distance between the array and the cavity grazing plane $r=13 \delta_{\omega_{0}}$, indicating that Strouhal numbers $S t_{D_{w}} \geq 3.32$ could be considered of acoustic nature prior to any filtering. This value corresponds roughly to the Strouhal number at which the $k-\omega, W T 1$ and $W T 2$ yield spectra identical to the original signal. Considering the pressure signal on a vertical line of 11 probes at $x=0.62 \mathrm{R}$ equally spaced from $z=2.7 \mathrm{H}$ to $z=4 \mathrm{H}$, the power spectral densities have been reduced using a normalization with an inverse power of the distance. To illustrate the far-field behavior, $P S D \times r^{2}$ 


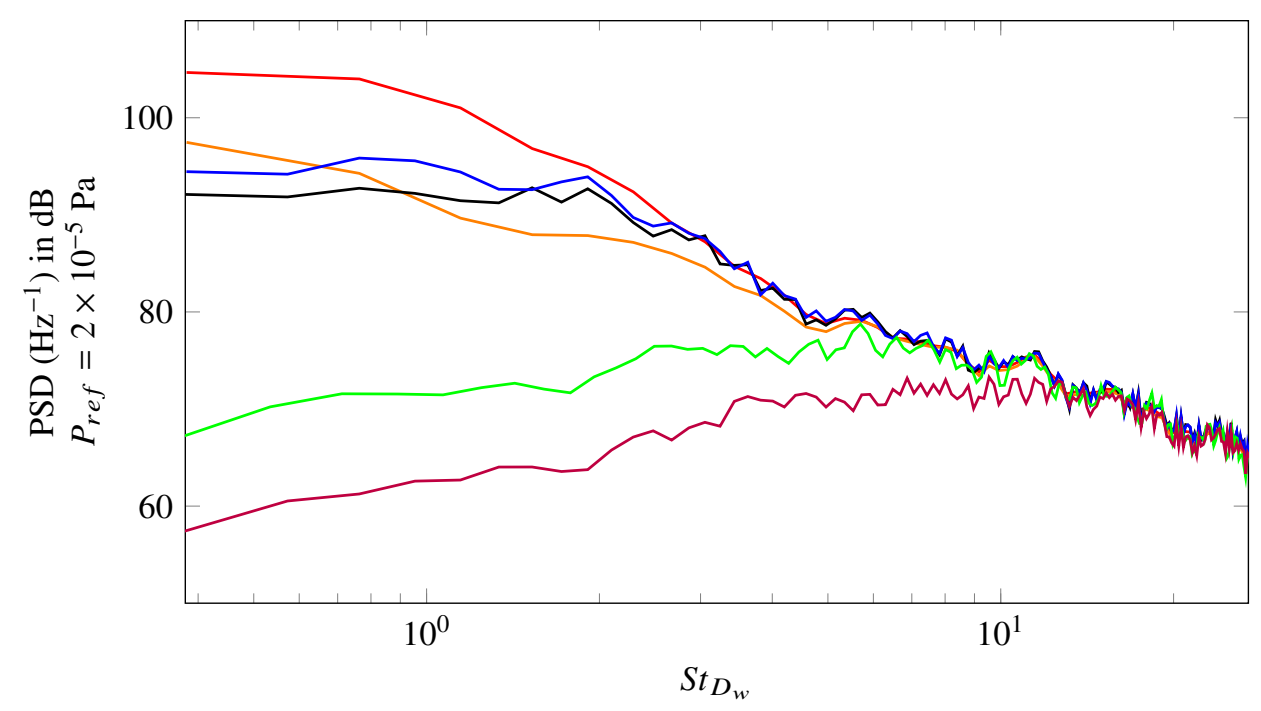

Fig. 12 Comparison of the filtering techniques at the microphone located at $x=0.62 R$ and $z=2.7 H$. (-) : Original signal, (-) : $k-\omega,(-): W T 1,(-): W T 2,(-): W T 3,(-): W T 4$

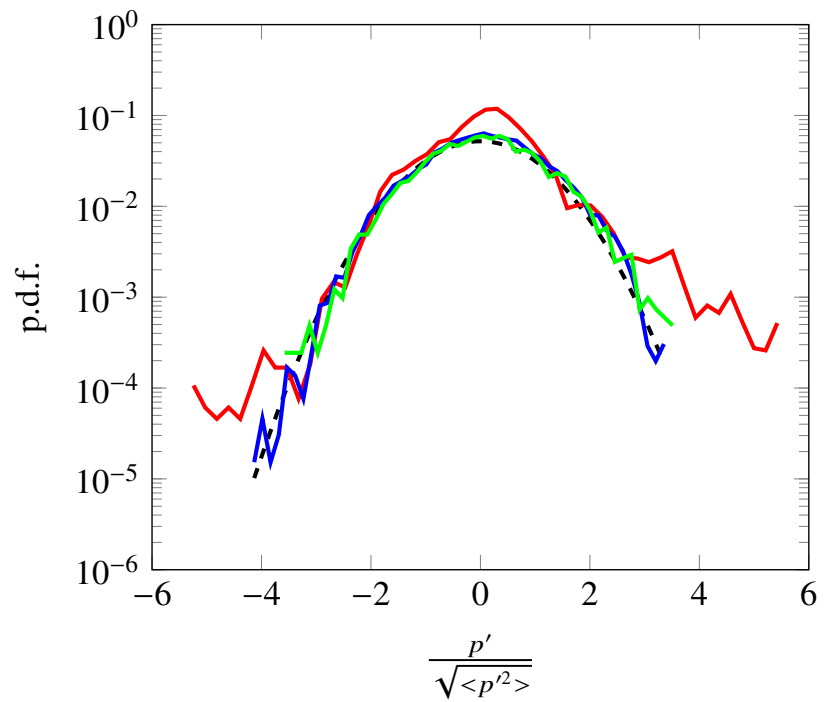

Fig. 13 PDF of (-) : original signal, (-) : WT2, (-) : WT3, (--) : Gaussian standard distribution

is plotted in figure 14a The spectra reduction are arbitrarily computed relatively to the nearest probe at $z=2.7 \mathrm{H}$ depicted in red. For comparison, the farthest probe located at $z=4 H$ is depicted in green. With this normalization, all spectra collapse at Strouhal numbers superior to the value of 3.32 previously predicted, confirming the $r^{-1}$ decay of the acoustic pressure for the highest frequencies. The spectra have also been reduced considering the $r^{-2}$ decay expected for the near-acoustic field [19] at distances such that $k_{0} r<1$ and the resulting spectra are shown in figure $14 \mathrm{~b}$ As the considered probe gets closer to the shear layer, this $r^{-2}$ behavior is less and less verified due to the growing amplitude of the pseudo-sound that rather scales with $r^{-3}$ and is thus dominant for the shortest distances [20]. The aim of this paragraph is to investigate the decay of the filtered pressure field with distance with the wavelet methods. To this end, the four wavelet separation methods have been employed at the 11 probes on the same vertical line and the resulting filtered component has been reduced using $r^{-1}$ and $r^{-2}$ distance normalization to check the ability of the wavelet methods to remove the pseudo-sound component while maintaining an acoustic behavior of the filtered pressure. The result of the PSD reductions with distance is presented in figure 15. While WT1 and WT2 restitute the $r^{-1}$ decay at high frequencies (figures $15 \mathrm{a} \mid 15 \mathrm{c}$ ), this behavior is less evident with WT3 (figure $15 \mathrm{e}$ ) and clearly not achieved with 


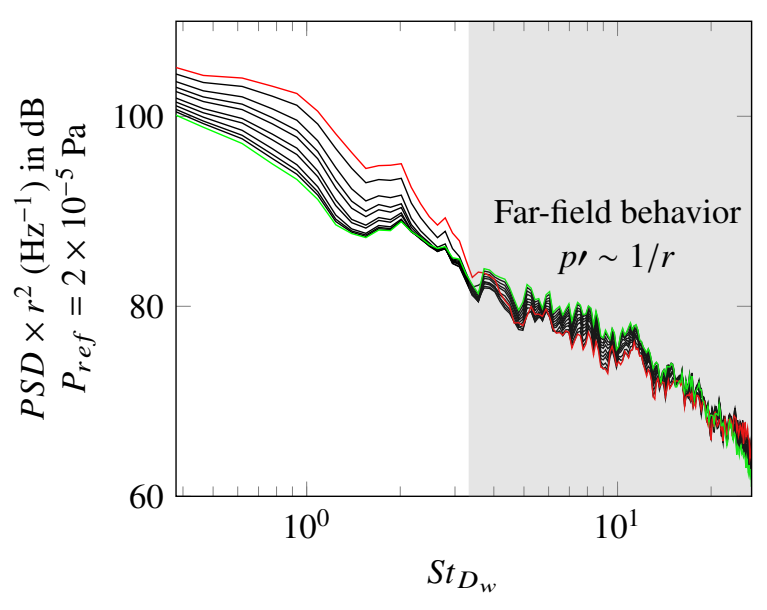

(a) $P S D \times r^{2}$

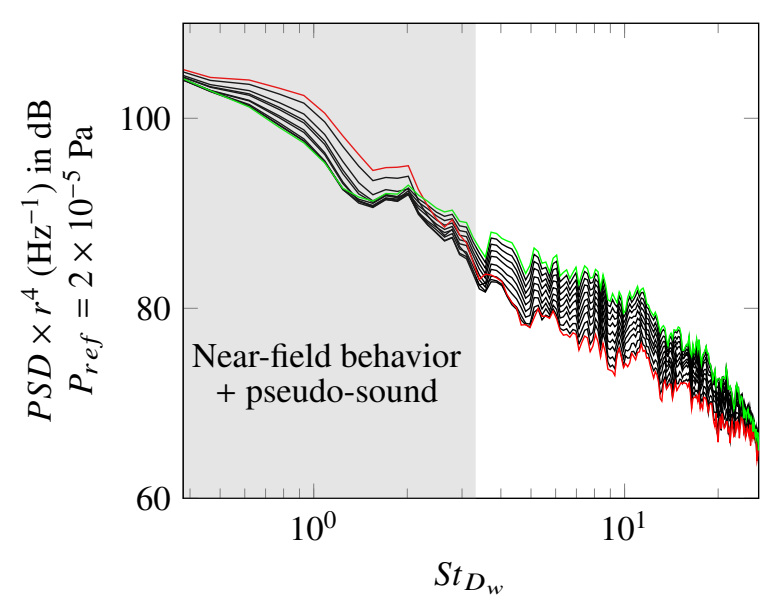

(b) $P S D \times r^{4}$

Fig. 14 Reduction of the original CFD pressure PSD with distance on a vertical line located at $x=0.62 R$ and $z$ varying between (-) $2.7 \mathrm{H}$ and (-) $4 \mathrm{H}$

WT4 for the closest point (figure $15 \mathrm{~g}$ ). As for the $r^{-2}$ decay, it is reasonably met with $W T 1$, except for two probes, but not for the three other methods, especially for $W T 3$ and $W T 4$ that already presented noise levels several decades lower than the classical $k-\omega$ filter in figure 12 .

\section{Conclusions}

The near pressure field of a landing gear subcomponent has been analyzed by means of wavelet-based separation algorithms recently proposed for the analysis of experimental turbulent jet noise data. The test case is a landing gear wheel isolated from the simplified geometry LAGOON. It consists in a circular cavity installed in a bluff body and can be regarded as a good compromise between academic cavities and more realistic landing gear geometries. While some analysis of the sound emission had already been performed on this case [9], the decomposition of the near pressure field had not been specifically addressed. As wavelets are gaining more and more interest in the aeroacoustic community and have proven efficient in the eduction of pseudo-sound for jet noise, the aim of this paper was to address their relevance on signals obtained from low Mach number compressible numerical simulations.

The more traditional $k-\omega$ Fourier filtering has been applied to enable comparisons between the methods. A notable difference between these two approaches resides in the fact that the $k-\omega$ requires a space-time description of the field (global nature), while the wavelet methods require local pressure signals. A well-known limitation of the $k-\omega$ approach is that low wavenumbers cannot be filtered due to the effective wavenumber resolution of the array $\Delta k$.

Overall, when looking at the signals filtered in the time domain, all the methods seem to efficiently remove the high-amplitude events related to the convection of turbulent eddies in the shear layer. The examination of their spectral representation in figure 12 shows that for $S t_{D w} \geq 15$, the filtered component is identical to the original signal for all methods. At lower Strouhal numbers, large differences are obtained between on one hand the $k-\omega, W T 1$ and WT2 methods that present levels greater by several decades to the low frequency component obtained with the WT3 and WT4 methods. This contrasts with the work of Mancinelli et al. [7] who obtained very similar spectra for the acoustic component with differences restricted to $1 \mathrm{~dB}$ for the whole frequency range they presented. It must be stressed that the Mach number of the present study is considerably lower than in their work, so the sound to source magnitude ratio might be in our case extremely weak, making the separation of the two contributions even more challenging.

The results obtained with the application of WT3 also indicate that the assumption of Gaussian nature for the acoustic pressure can be ambiguous when nonphysical high frequencies are present in the signal. Indeed, when performing a numerical simulation, one often has to maintain a low CFL number to ensure numerical stability and/or accurate propagation of the acoustic waves. This implies imposing a simulation sampling frequency that can easily reach values of the order of $10^{5} \mathrm{~Hz}$, while the mesh cutoff frequency is much lower. In this paper, we have presented the bandpass filter behavior of the scaled wavelets and shown in practice the importance of resampling the data to remove the 
Gaussian but nonphysical component of the signal.

While the Gaussian assumption seems reasonable for broadband noise associated with a shear layer, it might not be suited to problems such as cylinder noise where the acoustic field is typically cosine. Indeed, the probability density function of a cosine function has a typical $U$ shape and is therefore far from Gaussian, so classical Fourier analysis should be better suited to this class of problem. An application of these methods on the tandem cylinders test case by the authors, not reported here, confirms that the Gaussian white noise assumption can be a limitation to the tested methods when tonal noise is present.

Finally, an analysis of the filtered pressure field decay with distance has been carried out. It has been shown that, while WT1 and WT2 maintain the decay of the pressure field with the inverse of distance for the highest frequencies, the other methods do not present power spectral densities that collapse with such scaling. As for the lowest part of the spectrum, where large differences are found between the methods, none of them exhibits a clear decay with the square inverse of distance that could be expected.

These differences suggest perspectives for future work. Recalling that this near-field filtering is only a first step towards the use of causality methods to identify and understand the noise generating mechanisms, further research could focus on using the filtered signals as reference signals for methods such as Linear Stochastic Estimation as proposed by Grassucci et al. [14] to investigate the influence of the low frequency component and its link with other aerodynamic quantities.

\section{References}

[1] Tinney, C., and Jordan, P., "The near pressure field of co-axial subsonic jets," Journal of Fluid Mechanics, Vol. 611, 2008, pp. 175-204.

[2] Kerherve, F., Jordan, P., Cavalieri, A., Delville, J., Bogey, C., and Juvé, D., "Educing the source mechanism associated with downstream radiation in subsonic jets," Journal of Fluid Mechanics, Vol. 710, 2012, pp. 606-640.

[3] Druault, P., "Analysis of the acoustic/hydrodynamic separation in an aeroacoustic 2D plane mixing layer flow," $23 \mathrm{rd}$ AIAA/CEAS Aeroacoustics Conference, AIAA paper 2017-3000, 2017.

[4] Grizzi, S., and Camussi, R., "Wavelet analysis of near-field pressure fluctuations generated by a subsonic jet," Journal of Fluid Mechanics, Vol. 698, 2012, pp. 93-124.

[5] Mallat, S., A wavelet tour of signal processing, Elsevier, 1999.

[6] Farge, M., "Wavelet transforms and their applications to turbulence," Annual review of fluid mechanics, Vol. 24, No. 1, 1992, pp. 395-458.

[7] Mancinelli, M., Pagliaroli, T., Di Marco, A., Camussi, R., and Castelain, T., "Wavelet decomposition of hydrodynamic and acoustic pressures in the near field of the jet," Journal of Fluid Mechanics, Vol. 813, 2017, pp. 716-749.

[8] Manoha, E., Bulté, J., and Caruelle, B., "LAGOON: an experimental database for the validation of CFD/CAA methods for landing gear noise prediction," 14th AIAA/CEAS Aeroacoustics Conference (29th AIAA Aeroacoustics Conference), $2008, \mathrm{p}$. 2816.

[9] De La Puente, F., Sanders, L., Druault, P., and Vuillot, F., "Investigation on landing gear shallow round cavity flow field and noise signature," 22nd AIAA/CEAS Aeroacoustics Conference, AIAA paper 2016-2774, 2016.

[10] Gloerfelt, X., Bogey, C., Bailly, C., and Juvé, D., "Aerodynamic noise induced by laminar and turbulent boundary layers over rectangular cavities," 8th AIAA/CEAS Aeroacoustics conference $\mathcal{E}$ exhibit, 2002, p. 2476.

[11] Marsden, O., Bogey, C., and Bailly, C., "Investigation of Flow Features Around Shallow Round Cavities Subject to Subsonic Grazing Flow," Physics of Fluids, Vol. 24, No. 12, 2012, p. 125107.

[12] Ruppert-Felsot, J., Farge, M., and Petitjeans, P., "Wavelet tools to study intermittency: application to vortex bursting," Journal of Fluid Mechanics, Vol. 636, 2009, pp. 427-453.

[13] Donoho, D. L., and Johnstone, J. M., "Ideal spatial adaptation by wavelet shrinkage," Biometrika, Vol. 81, No. 3, 1994, pp. $425-455$.

[14] Grassucci, D., Camussi, R., Jordan, P., and Grizzi, S., "Intermittency of the near pressure field induced by a compressible coaxial jet," Experiments in Fluids, Vol. 56, No. 2, 2015, p. 23. 
[15] Pérez Arroyo, C., Daviller, G., Puigt, G., Airiau, C., and Moreau, S., "Identification of temporal and spatial signatures of broadband shock-associated noise," Shock Waves, Vol. 29, No. 1, 2019, pp. 117-134. doi:10.1007/s00193-018-0806-4.

[16] Kœnig, M., Cavalieri, A. V., Jordan, P., Delville, J., Gervais, Y., and Papamoschou, D., "Farfield filtering and source imaging of subsonic jet noise," Journal of Sound and Vibration, Vol. 332, No. 18, 2013, pp. 4067-4088.

[17] Camussi, R., Di Marco, A., and Castelain, T., "Statistical analysis of the hydrodynamic pressure in the near field of compressible jets," International Journal of Heat and Fluid Flow, Vol. 64, 2017, pp. 1-9.

[18] Crighton, D. G., Dowling, A. P., Williams, J. E. F., Heckl, M., and Leppington, F. G., Matched Asymptotic Expansions Applied to Acoustics, Springer London, London, 1992, pp. 168-208. doi:10.1007/978-1-4471-0399-8_6.

[19] Jacob, M. C., "Introduction to experimental aeroacoustics," VKI Lecture Series, Von Karmán Institute for Fluid Dynamics, 2017.

[20] Ribner, H. S., "Aerodynamic sound from fluid dilatations; a theory of the sound from jets and other flows," Tech. rep., University of Toronto, 1962. 


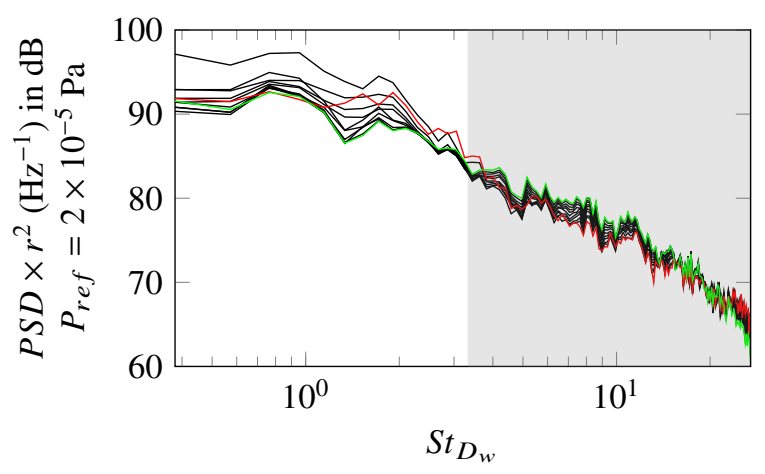

(a) $W T 1: P S D \times r^{2}$

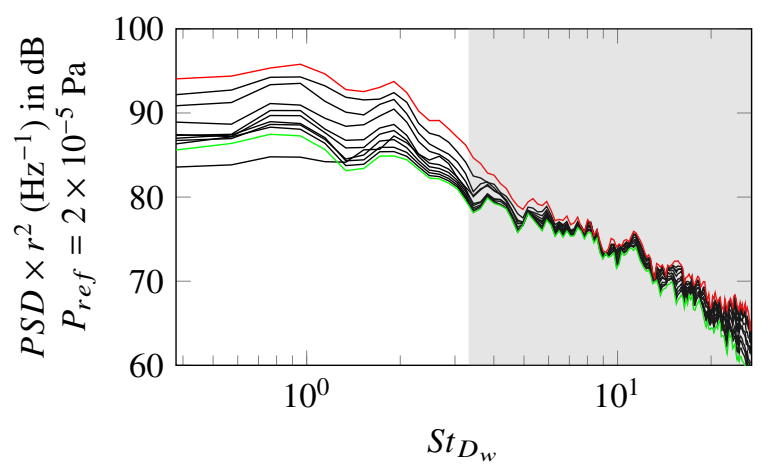

(c) $W T 2: P S D \times r^{2}$

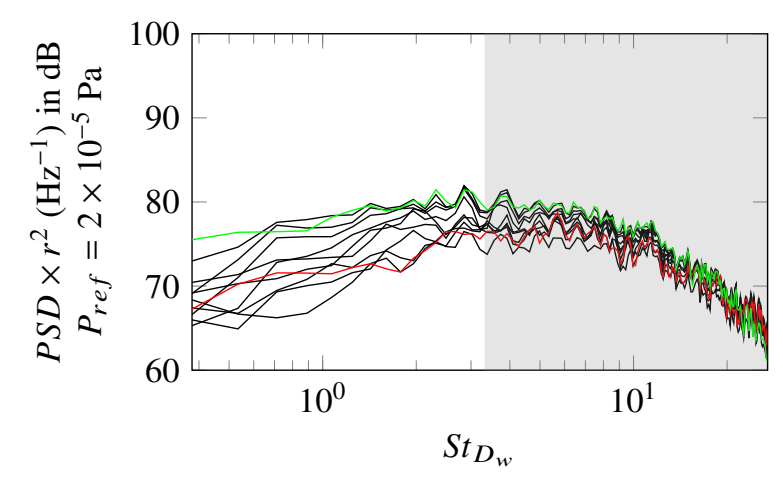

(e) $W T 3: P S D \times r^{2}$

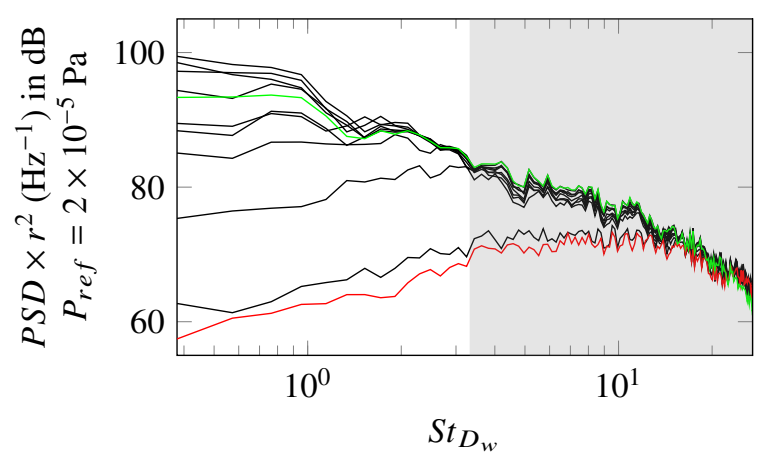

(g) WT4 : $P S D \times r^{2}$

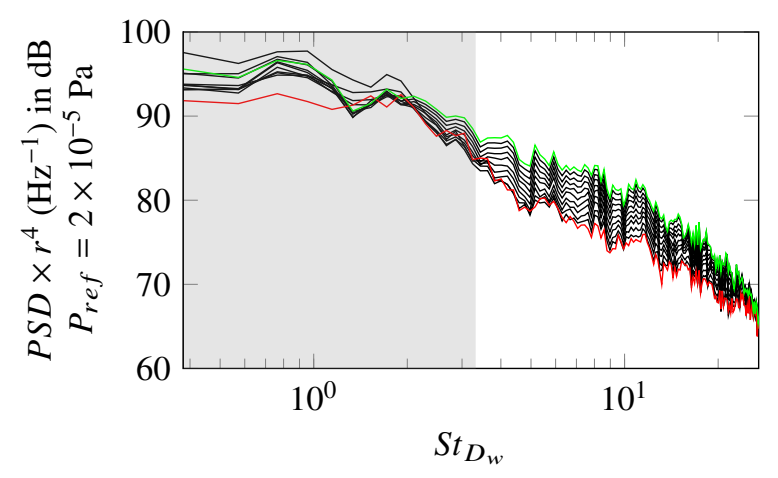

(b) $W T 1: P S D \times r^{4}$

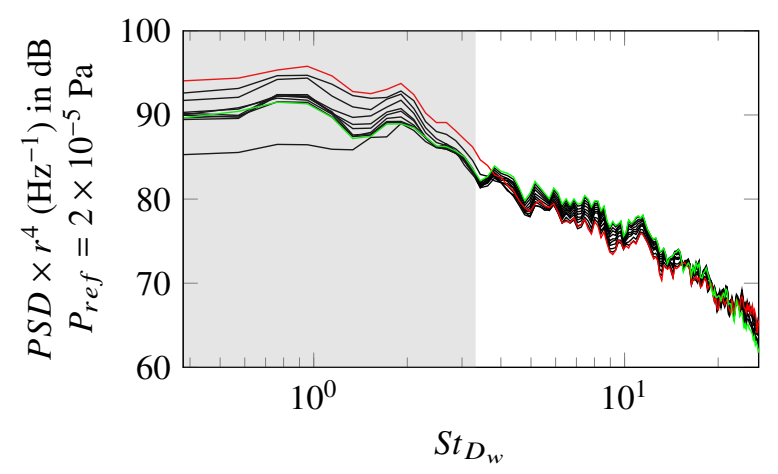

(d) $W T 2: P S D \times r^{4}$

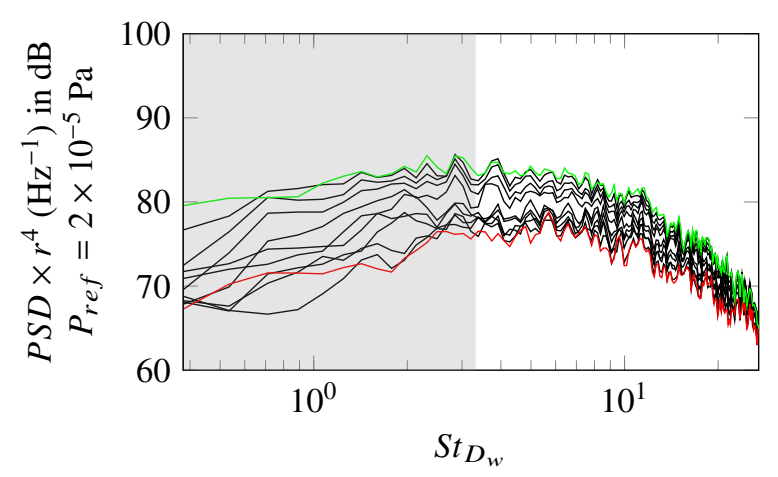

(f) $W T 3: P S D \times r^{4}$

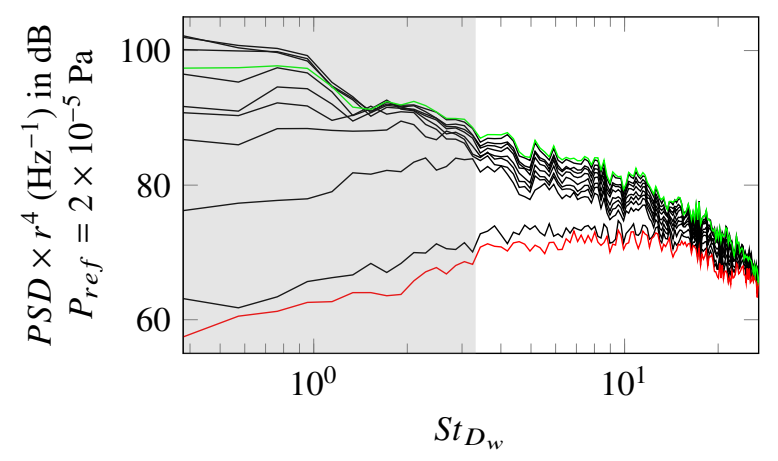

(h) WT4 : PSD $\times r^{4}$

Fig. 15 Reduction of the filtered pressure PSD with distance on a vertical line located at $x=0.62 R$ and $z$ varying between (-) $2.7 \mathrm{H}$ and (-) $4 \mathrm{H}$ 